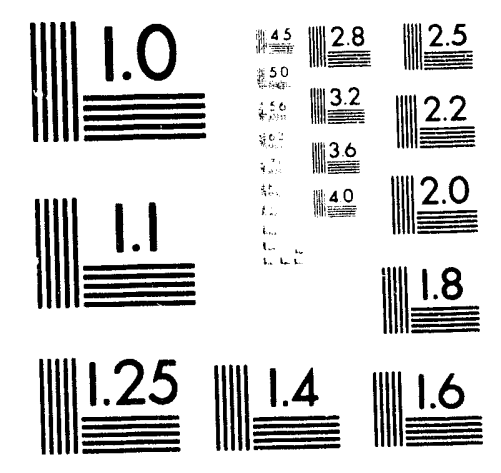



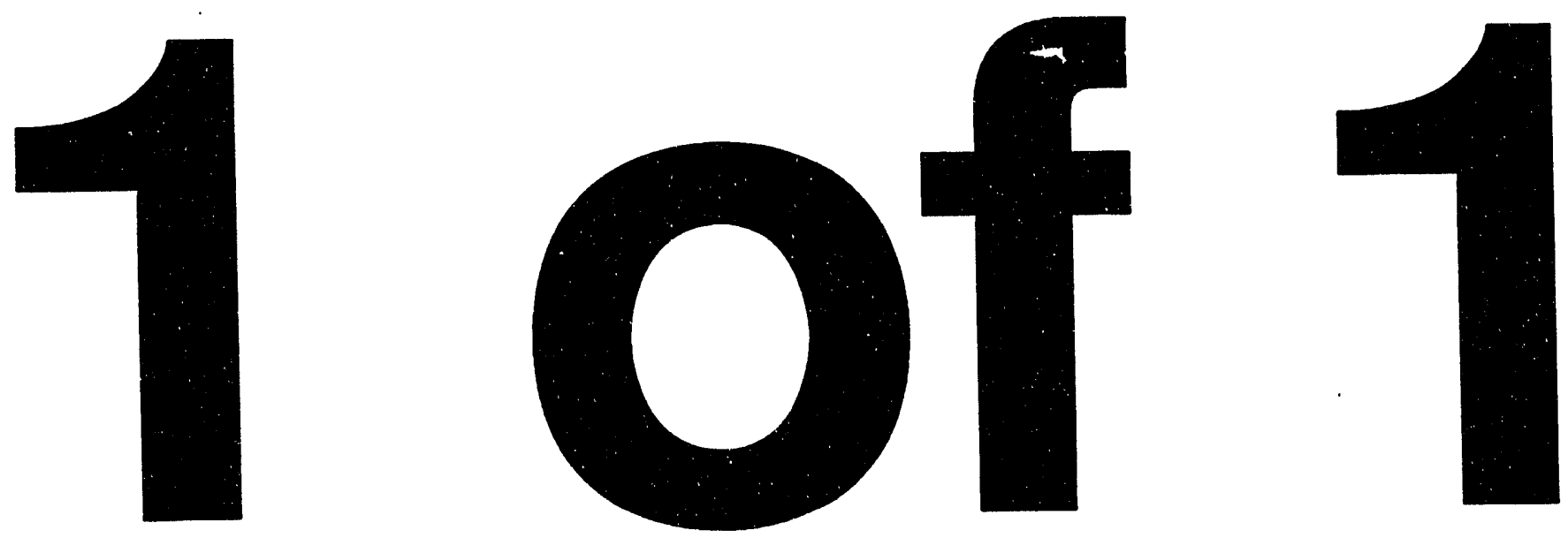


\section{LA-UR $9 \ddot{3}-2660$}

TITLE: STUDIES OF CLAYS AND CLAY MINERALS USING X-RAY POWDER DIFFRACTION AND THE RIETVELD METHOD

AUTHOR(S): David L. Bish

SUBMITTED TO: Clay Minerals Society

Short Course on Computer Applications in Clay Mineralogy

San Diego, CA

September 25, 1993

\section{DISCLAIMER}

This report was prepared as an account of work sponsored by an agency of the United States Government. Neither the United States Gnvernment nor any agency thereof, nor any of their employees, makes any warranty, express or implied, or assumes any legal liability or responsibility for the accuracy, completeness, or usefulness of any information, apparatus, product, or process disclosed, or represents that its use would not infringe privately owned rights. Reference herein to any specific commercial product, process, or service by trade name, trademark, manufacturer, or otherwise does not necessarily constitute or imply its endorsement, recommendation, or favoring by the United States Government or any agency thereof. The views and opinions of authors expressed herein do not necessarily state or reflect those of the United States Government or any agency thereof.

By acceptance of this articie, the publisher recognizes that the U.S. Government retains a nonexclustve, royalty-free Ilcense to publtsh or reproduce the published form of this contivition, or to allow othe to do so, for U.S. Government purposes.

The Los Alamos National Laboratory requests that the putblisher Identity this artlde as work pertormed under the ausplces of the U.S. Department of Energy.

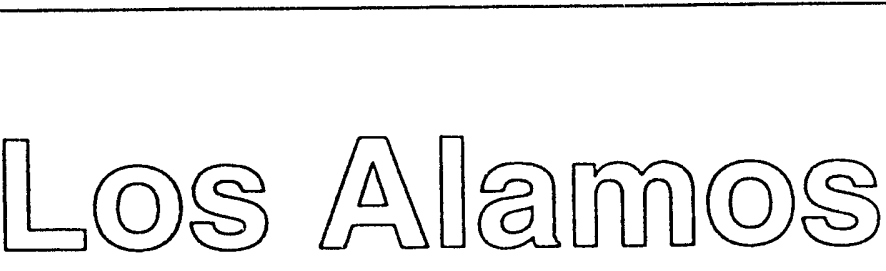

$$
\text { FASTR }
$$

Los Alamos National Laboratory Los Alamos, New Mexico 87545 


\title{
STUDIES OF CLAYS AND CLAY MINERALS USING X-RAY POWDER DIFFRACTION AND THE RIETVELD METHOD
}

\author{
David L. Bish
}

\section{CONTENTS}

Introduction

The Rietveld Method

Starting Models for Refinement

Transmission electron microscopy

Distance least-squares modeling

Electrostatic energy minimization

Disorder In Clays And Clay Minerals

Applications Of The Rietveld Method To Clay Minerals

Crystal Structure Refinements

Dickite

Kaolinite

Chlorite

93

Partial Structure Solution

Hydrogen Atoms in Kaolinite

Hydrogen Atoms in Dickite

Interlayer Structure of Kaolinite Intercalates

Exchangeable Cations and Water in Sepiolite

Quantitative Analysis

100

Theory

100

Application

104

Refinement of Unit-Cell Parameters

106

Analysis of Peak Broadening

108

Goethite

Sample Refinement

112

Sample Preparation and Data Collection $\quad 112$

Refinement Strategies

Conclusions

Acknowledgments

References Cited 


\title{
STUDIES OF CLAYS AND CLAY MINERALS USING X-RAY POWDER DIFFRACTION AND THE RIETVELD METHOD
}

\author{
David L. Bish \\ Earth and Environmental Sciences \\ Los Alamos National Laboratory \\ Los Alamos, New Mexico 87545
}

\section{INTRODUCTION}

The Rietveld method was originally developed (Rietveld, 1967, 1969) to refine crystal structures using neutron powder diffraction data. Since then, the method has been increasingly used with X-ray powder diffraction data, and today it is safe to say that this is the most common application of the method. The method has been applied to numerous natural and synthetic materials, most of which do not usually form crystals large enough for study with single-crystal techniques. It is the ability to study the structures of materials for which sufficiently large single crystals do not exist that makes the method so powerful and popular. It would thus appear that the method is ideal for studying clays and clay minerals. In many cases this is true, but the assumptions implicit in the method and the disordered nature of many clay minerals can limit titsapplicability. This chapter will describe the Rietveld method, emphasizing the assumptions important for the study of disordered materials, and it will outline the potential applications of the method to these minerals. These applications include, in addition to the refinement of crystal structures, quantitative analysis of multicomponent mixtures, analysis of peak broadening, partial structure solution, and refinement of unit-cell parameters.

As: important requirement with the Rietveld method, and one that is often only assumed to be met, is that the diffraction pattern must exhibit only Bragg diffraction effects. Unfortunately, the layered n'ture of many clay mineral structures makes them prone to stacking disorder, giving rise to two-dimensional diffraction effects. This aspect of their structures makes the application of the Rietveld method to clay minerals difficult and, in many cases, unwarranted. Thus it is critical to understand the limitations of the method when applying it to poorly ordered materials. In particular, it is important to recognize that two-dimensional diffraction bands cannot be simulated simply by broadening the Bragg reflections.

In spite of these limitations, the Rietveld method has been successfully applied to several well-ordered clays, including kaolinite, dickite, nacrite, chlorite, and the chain-structure clay mineral sepiolite. In these cases, samples were specially chosen which showed little or no evidence of two-dimensional diffraction effects. These studies yielded detailed structural information, including positions of exchangeable cations, data on octahedral and tetrahedral cation ordering, and the detailed effects of temperature on the structures. For well-ordered materials such as these, partial structure solution is an important adjunct to Rietveld refinement, usually using 
difference-Fourier syntheses. The coupled use of these two procedures has been shown to be very effective in locating $\mathrm{H}$ atoms (using neutron diffraction data) and interlayer intercalate species such as hydrazine and $\mathrm{H}_{2} \mathrm{O}$ molecules in kaolin minerals. Application of the Rietveld method to a chlorite with semi-random stacking (i.e., some two-dimensional diffraction effects) illustrates some of the problems inherent with disordered materials. The refinement yielded precise cell parameters, but atomic positions and occupancies were artifacts due to the $b / 3$ layer shifts.

Use of the Rietveld method with non-layer-structure clay minerals has been fruitful, as these materials are not usually hampered by two-dimensional diffraction effects, although they are often very poorly crystalline (i.e., they have very small crystallite sizes). For example, several studies of goethite $(\mathrm{FeOOH})$ have elucidated the detailed structural changes occurring with isomorphous substitutions of $\mathrm{Al}, \mathrm{Cr}$, and $\mathrm{Mn}$ for $\mathrm{Fe}$; other studies have identified the sources of the pronounced peak broadening which appear to depend partially on these isomorphous substitutions. Reflections in Mn-substituted goethites are predominantly strain broadened, due to Jahn-Teller distorted octahedra, whereas Al-substituted goethites exhibit primarily crystallite-size broadening (Bish and Ebinger, 1989). Even with very broad reflections, the Rietveld method appears to give useful structural information, as long as diffraction data show no non-Bragg diffraction effects.

\section{THE RIETVELD METHOD}

Conceptually, the Rietveld method is different from other more traditional techniques for evaluating $X$-ray powder diffraction data in that a refinement uses digital diffraction data and is done on a point-by-point (step-by-step) basis instead of dealing with individual reflections. Specifically, a Rietveld refinement minimizes $R$, the sum of the weighted, squared differences between observed and calculated intensities at every $2 \theta$ step in a digital powder pattern,

$$
R=\sum_{i} w_{i}\left|y_{i}(o)-y_{i}(c)\right|^{2}
$$

where $y_{i}(0)$ and $y_{i}(c)$ are observed and calculated intensities at point $i$, and $w_{i}$ is the weight assigned to each intensity. The calculated intensities at each point (or $2 \theta$ step), $y_{i}(c)$, are determined by summing the contributions from background and all neighboring Bragg reflections as:

$$
y_{i}(c)=S \sum_{k}\left(p_{k} L_{k}\left|F_{k}\right| G\left(\Delta \theta_{i k}\right) P_{k}\right)+y_{i b}(c)
$$

where $S$ is a phase-specific scale factor, $p k$ is the multiplicity factor, $L_{k}$ is the Lorentz and polarization factor for the $k$ th reflection, $F_{k}$ is the structure factor for an individual reflection for a particular phase, $G\left(\Delta \theta_{i k}\right)$ is a reflection profile function, $\theta_{i k}$ is the Bragg angle for the $k$ th reflection, $P_{k}$ is a preferred orientation function, and $y_{i} b(c)$ is a refined background. 
Equation (2) clearly illustrates a very important aspect of Rietveld refinements, namely that the method assumes the presence of Bragg diffraction and the absence of non-Bragg diffraction effects. Thus, only integral values of $h, k$, and $l$ are allowed and the structure factor cannot vary actoss a reflection. The latter is important with poorly crystalline materials, and the Rietveld method cannot be rigorously applied in these cases because it assumes a given structure factor for each reflection. In addition, because of these factors, samples ideally must not possess any layerstacking disorder or turbostratic stacking. These aspects of Rietveld refinements are of utmost importance when considering applications to clays and clay minerals, and unfortunately these requirements rule out most clay minerals.

In the absence of two-dimensional diffraction effects, preferred orientation of crystallites is typically the most significant problem encountered in a refinement. Because preferreci orientation is such an important consideration when dealing with many clays and clay minerals, it is worthwhile to describe the methods of correcting for this effect. The first popular analytical treatment of preferred orientation in the Rietveld method was the use of the correction factor, $G_{h k l}$ (Rietveld, 1969),

$$
G_{\mu L}=\exp \left(-G_{1} \alpha^{2}\right)
$$

where $\alpha$ is the acute angle between the normal to the crystallites and the scattering vector. $G_{I}$ is a refinable parameter and is a measure of the half-width of the Gaussian distribution of the normals about the preferred orientation direction. This formulation has met with some success with neutron diffraction data and was slightly modified by Toraya and Marumo (1981) to yield a correction factor $\left(G_{h k l}\right)$ of the form

$$
G_{h e l}=G_{2}+\left(1-G_{2}\right) \exp \left(-G_{1} \alpha^{2)}\right)
$$

where $G_{l}$ and $\alpha$ are analogous to $G_{l}$ and $\alpha$ in Eq. (3) and $G_{2}$ is the (refinable) fraction of oriented crystallites.

Neither Eq. (3) nor (4) has been very successful with X-ray powder diffraction data, for which preferred orientation is usually a greater problem. In an attempt to rectify this situation, Dollase (1986) examined a variety of analytical forms previously proposed to represent pole-figure profiles. Dollase concluded that the March (1932) function, which describes the pole-density distribution yielded by rigid-body rotation of inequant crystallites upon axially symmetric volumeconserving expansion or compression, has significant advantages over other proposed functions. The procedure used in a Rietveld refinement with the March function correction consists of fitting the pole-density profile using a function with a single variable parameter. The function used by Dollase (1986) is 


$$
G_{k+1}=\left(r^{2} \cos ^{2} \alpha+r^{-1} \sin ^{2} \alpha\right)^{-3 / 2}
$$

where $\alpha$ is as defined for Eq. (3) and $r$ is the only adjustable coefficient related to the degree of preferred orientation. Dollase also pointed out that most of the earlier preferred orientation correction functions work well with weakly developed preferred orientation, such as is common with non-platy minerals in a neutron diffraction experiment, but they do not work well with typical materials in an X-ray diffraction experiment. Moreover, an important shortcoming of the previous corrections [e.g., Eq. (3) or (4)] is that none is normalized to unit integral which means that any change in a refinable parameter will be counterbalanced by a change in the overall scale factor. This property of the correction is particularly important for quantitative analysis using the Rietveld method. The author's experience has shown that the March function suggested by Dollase works well for low to high degrees of preferred orientation in platy materials and fairly well for rod-like materials with low degrees of preferred orientation. Many of the popular Rietveld computer programs now include the March function for preferred orientation corrections.

\section{Starting Models for Refinement}

By its very nature, structure refinement relies on the existence of a reasonably accurate starting structure model. The very large number of published refined structures available in the literature provides a starting point for most structure refinements. Diffraction data are then used to improve, or to refine, the model to yield optimum agreement between observed and calculated observations. In addition, geometrical constraints and crystal chemical intuition have played a part in solving structures. However, as the complexity of structures increases, additional information is usually required and can often be obtained from transmission electron microscope (TEM) images, distance least-squares (DLS) modeling, and/or energy minimizations (Bish, 1992).

Transmission electron microscopy. Additional structural data have come, in recent years, in the form of high-resolution transmission electron microscopy (HRTEM) images, which have been used to develop structure models (e.g., Banfield et al., 1991). The structure models for several cases were derived from HRTEM images which were then further optimized using DLS modeling to develop useful starting models for Rietveld refinement. Within the past twenty years, transmission electron microscopy has advanced to the point where it can produce images of the atomic arrangement in a crystal, often easily yielding insights into general structural schemes and, in some cases, solving apparently intractable problems (e.g., Veblen, 1985; Buseck and Veblen, 1988). It is important to note, however, that HRTEM images must be used with caution. Minor changes in focus in the microscope can cause large changes in the high-resolution image, greatly changing the images (e.g., Guthrie and Veblen, 1990). In order to interpret the images unambiguously, it is necessary to couple HRTEM observations with modeling of the images using a multi-slice calculation; such calculations require at least an approximate knowledge of the structure (e.g., O'Keefe, 1984; Post and Veblen, 1990). 
Bish

Distance least-squares modeling. DLS modeling (Meier and Villiger, 1969) is a powerful method for determining whether or not a paricular model is geometrically possible. The method is similar in many respects to conventional crystal structure refinement, particularly in that it requires a starting model. These models can be obtained by analogy with other structures or by crystal chemical reasoning (or from HRTEM images). Fortunately, the DLS method is very robust and calculations will usually converge, even given very poor starting models. An optimum distance model is obtained by varying atomic coordinates and unit-cell parameters to minimize discrepancies between interatomic distances calculated for the model structure and prescribed (input) distances that comprise the observations. Input distances are usually obtained from published, well-refined analogous structures and can often be estimated to within several hundredths of an $\AA$. Weights applied to distances are usually related either to Pauling bond strengths or to some empirical measurement, such as stretching force constants; weights based on the latter emphasize shorter distances and those involving highly charged ions much more strongly than the former method. Known unit-cell parameters can be used in many cases to constrain the refinements further, resulting in more reasonable structures. Unit-cell parameters can usually be obtained by indexing an X-ray powder or electron diffraction pattern using well-established techniques when beginning with unknown or pooriy known structures.

DLS modeling of a completely unknown structure requires some imagination and adjunct information (such as HRTEM images), but modeling a variant of a known material can be quite easy. For example, if an intercalation reaction in a layered material such as kaolinite, $\mathrm{Al}_{2} \mathrm{Si}_{2} \mathrm{O}_{5}(\mathrm{OH})_{4}$, gives rise to a larger unit repeat in the direction perpendicular to the layers, DLS modeling can use the parent structure together with the new unit-cell parameters to represent the new structure. Of course, one must either provide unit-cell parameters or use distance observations sufficient to constrain the structure in three dimensions. After any DLS modeling, calculated diffraction pattems usually provide a quick idea of how reasonable the structure model is, easily revealing whether the symmetry and cell are correct and how closely the model structure approximates the real one. This procedure is similar to that used by Banfield et al. (1991) with HRTEM images, namely obtaining a model structure and determining how well observations agree with those calculated based on the model. In some cases, a DLS model structure can also minimize the problems of false-minima in a Rietveld refinement, as appears to be the case for one study of kaolinite (see discussion in Bish and Von Dreele, 1989).

Electrostatic energy minimization. Electrostatic energy minimization is a final method that is proving increasingly useful in supporting determinations of H-atom orientations and extraframework species positions. This method combines calculation of the Coulomb portion of the lattice energy with some formulation of the short-range repulsive energies to determine the minimum-energy configuration of a particular arrangement of atoms. Guthrie and Bish (1991) applied this method to a study of the $\mathrm{H}$ orientations in kaolinite, for which $\mathrm{H}$ positions had just recently been determined using Rietveld refinement/difference-Fourier $(\Delta F)$ methods and neutron powder diffraction data. It is very interesting that the results of the electrostatic minimizations reproduced both the $\mathrm{H}$ positions and the general shape of the potential wells within which the $\mathrm{H}$ atoms resided. Thus, this method explained and supported the anisotropic displacement parameters of the $\mathrm{H}$ atoms observed in the Rietveld refinement. Giese (1982) and Guthrie and Bish (1991) also used these methods to predict the $\mathrm{H}$ positions and orientations in layer silicates for which complete structure refinements have not been performed. Catlow et al. (1986) described 
numerous uses of electrostatic methods, including studying cation distributions in zeolites, and they commented on the possibilities of coupling energy minimizations with structure refinements using diffraction data.

\section{DISORDER IN CLAYS AND CLAY MINERALS}

Clay minerals exhibit a wide range of types of order/disorder, some of which affect the applicability of the the Rietveld method (see Brindley, 1980, for a general discussion). Substitutional disorder, such as the occupancy of a crystallographically distinct site by more than one type of atom, is very common in minerals. Examples of such disorder include the tetrahedral $\mathrm{Al} / \mathrm{Si}$ distribution in $2 M_{I}$ micas and $\mathrm{Fe} / \mathrm{Al}$ distribution on the octahedral site in goethite. In general, this type of disorder does not affect the diffraction pattern in a way that would limit the applicability of the Rietveld method. However, substitutional disorder can give rise to so-called lattice microstrain, as a result of the occupation of crystallographically "equivalent" sites by significantly different cations (or anions). An example of such an effect are the Mn-substituted goethites, in which octahedra occupied by $\mathrm{Mn}$ are significantly more distorted than those occupied by $\mathrm{Fe}$, producing a crystallite containing unit cells of different dimensions.

Strain broadening contrasts with that produced by the presence of very small crystallite sizes in a sample. Reflections are broadened by the presence of small crystallite sizes in proportion to the inverse of the crystallite size in a particular direction related to the reflection. For example, decreasing the packet thickness in a clay mineral will result in broadening of all reflections with a non-zero $l$ Miller index. Reflections with an $l$ Miller index of zero will be unaffected, all other factors remaining the same. Both strain and crystallite-size reflection broadening can be considered in the profile shape model and thus can be modeled explicitly in a Rietveld refinement. The Rietveld code GSAS (Larson and Von Dreele, 1988) can also model anisotropic crystallite size and strain broadening, in which different classes of reflections are broadened differently by both factors.

An additional type of disorder that is very common with clay minerals is layer stacking disorder. This type of disorder has been described in detail by Reynolds (1989) and only a summary will be provided here. There are several types of disorder common with clays and clay minerals, and perhaps the first step in a hypothetical sequence from ordered to disordered materials is so-called semi-random stacking. Semi-random stacking is seen most often with chlorites, in which the 2:1 layers above and below the interlayer hydroxyl sheet maintain hydrogen bond contact by adopting positions related by $\pm b / 3$. At each interface above and below the interlayer sheet, three positions are available, and the layers on either side of the interface adopt one of these positions randomly. Because the layers "choose" randomly from among a limited number of choices, the resulting structures are termed "semi-random." The layer shifts are all related by $\pm b / 3$ irrespective of which position is chosen. Thus, reflections with indices in which $k \neq 3 n$ will be streaked, producing a two-dimensional diffraction band.

If individual layers or packets of layers are randomly rotated or displaced in the $X-Y$ plane but the repeat is maintained along $Z$, a turbostratic structure results. Such a structure is much like a 
Bish

deck of playing cards that has been randomly rotated and displaced so that the cards are not lined up. In this case, the crystallites scatter as single layers and the only dimensional repeat is along $Z$. Therefore, only $00 l$ Bragg reflections will occur, and the remaining $h k l$ reflections will be smeared out into two-dimensional diffraction bands. In reciprocal space, the reciprocal lattice nodes that occur for an ordered crystal degenerate into a set of rods along $c *$. The intensity varies continuously along these rods, and the breadth or circumference of the rods depends upon the crystal perfection in the $X-Y$ plane (Figure 1). Reynolds (1989) described several methods of calculating the intensity distribution diffracted from a powder of such material, and he showed that such calculations depend on the type of layer stacking defects (simple rotations, shifts, and/or layer separations) and the statistical distribution of such defects. Clearly, such a calculation is not reproduced by the mathematics in Eq. (2), and this equation does not in any way model twodimensional diffraction effects. Figure 2, comparing observed kaolirite and halloysite diffraction patterns with calculated kaolinite patterns broadened using the profile shape function, further reinforces this concept and shows that simple broadening in no way models the two-dimensional diffraction effects in the diffraction pattem of halloysite.

A final step toward disordered materials is a material possessing little or no long-range threedimensional order, that is, an amorphous or glassy material. Reynolds (1989) presented a brief overview of diffraction from such materials; suffice it to say that, as with two-dimensional diffraction effects, diffraction effects from an amorphous material, cannot be modeled by the Rietveld method although some programs have the capability to model the "amorphous humps" as background.

\section{Effects of Disorder on Diffraction Patterns}

The effects of isotropic or anisotropic crystallite size and/or strain broadening are to broaden some or all reflections in a diffraction pattern, and these effects can be reasonably well modeled and distinguished with the Rietveld method. Bish and Ebinger (1989) showed for goethite that it can be fallacious to assume that broad reflections are always due to small crystallite size effects, as is implicitly assumed when applying the Scherrer equation. Strain broadening effects are not often identified in clays and clay minerals but they are probably more common than realized, as a result of substitutional and displacive defects.

Virtually all layer silicates exhibit at least some layer starking disorder due to relatively weak forces that hold the layers together. The results of Walker and Bish (1992) for a $\mathbb{\Pi b} \mathrm{Mg}$-chamosite show this effect and also illustrate some of the pitfalls of attempting a structure refinement using data exhibiting some non-Bragg diffraction effects (Figure 3). Note in Figure $3 \mathrm{~b}$ that the calculated pattern represents the observed pattern reasonably well, but in Figure $3 \mathrm{a}$, there is very poor agreement between observed and calculated data, and only a broad elevation of background is present with no discrete peaks. Obviously, attempting to refine a crystal structure using such data will result in an artifact, due to the absence of considerable diffraction information (i.e., discrete $k$ $\neq 3 n$ reflections) 


\section{Studies of Clays Using the Reitveld Method}

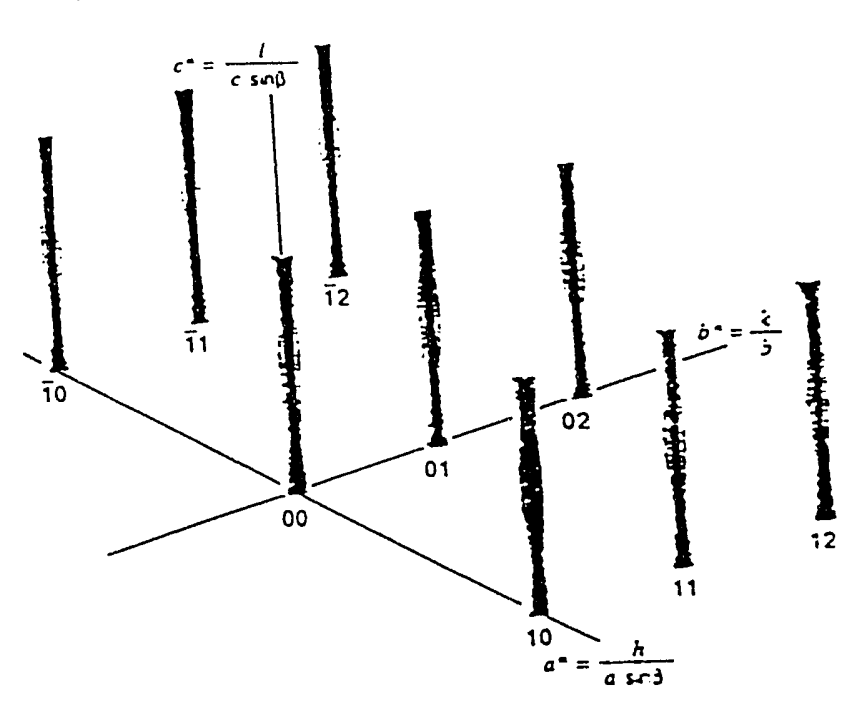

Figure 1. Schematic model of the reciprocal lattice of a turtostratically stacked layer silicate. Note that the reciprocal lattice nodes have degenerated into rods along ${ }^{*}$ and the intensity along these rods varies as a function of $l$.

Turbostratic stacking, or the absence of any significant long-range order in the $Z$ direction, occurs in essentially all smectites, most vermiculites, and many other $2: 1$ and $1: 1$ layer silicates. As described above, this type of stacking results in the loss of most or all three-dimensional diffraction information, and diffraction patterns consist of broadened $00 \mathrm{l}$ reflections and twodimensional diffraction bands. Figure 4 shows the features expected from a turbostratically stacked layer silicate, in this case Na-SWy-1 smectite, with broadened $00 \mathrm{l}$ reflections and distinct two-dimensional diffraction bands. Figure $5 \mathrm{a}$ and $\mathrm{b}$, showing unground and ground dickite respectively, illustrate further the difficulties encountered with two-dimensional diffraction bands. Note that the observed data in Figure 5 a can be modeled well, but the data in Figure 4 and Figure $5 \mathrm{~b}$ cannot be modeled correctly or completely using the Rierveld method, because there is no way of correctly modeling the broad, asymmetric bands. However, it is possible to perform a onedimensional refinement, using only the $00 \mathrm{l}$ data and refining only those parameters that define the structure in the $Z$ direction (e.g., $c$ unit-cell parameter, $z$ atom parameters, profile parameters for the $00 l$ reflections). A significant difficulty with this methodology would be correctly subtracting the intensity contribution of two-dimensional diffraction bands to the overlapping $00 \mathrm{l}$ reflections at higher angles unless a highly oriented sample were used. The application of the Rietveld method to these minerals will be severely limited because most clays exhibit at least some layer-stacking disorder that gives rise to two-dimensional diffraction effects. However, it is important to emphasize that the application of any diffraction method to disordered materials is difficult. For example, there are numerous cases in the literature where a partially disordered crystal was examined using single-crystal methods, and a conventional least-squares refinement was conducted using diffraction data collected from the crystal. It is common in such cases to obtain data at the theoretical reciprocal lattice nodes, correcting for the continuous or semi-continuous background around the nodes, and subsequently to ignore the diffuse scattering between reciprocal lattice nodes. This method is no more correct than the application of the Rietveld method to a diffraction pattem exhibiting two-dimensional diffraction effects. 


\section{Bish}

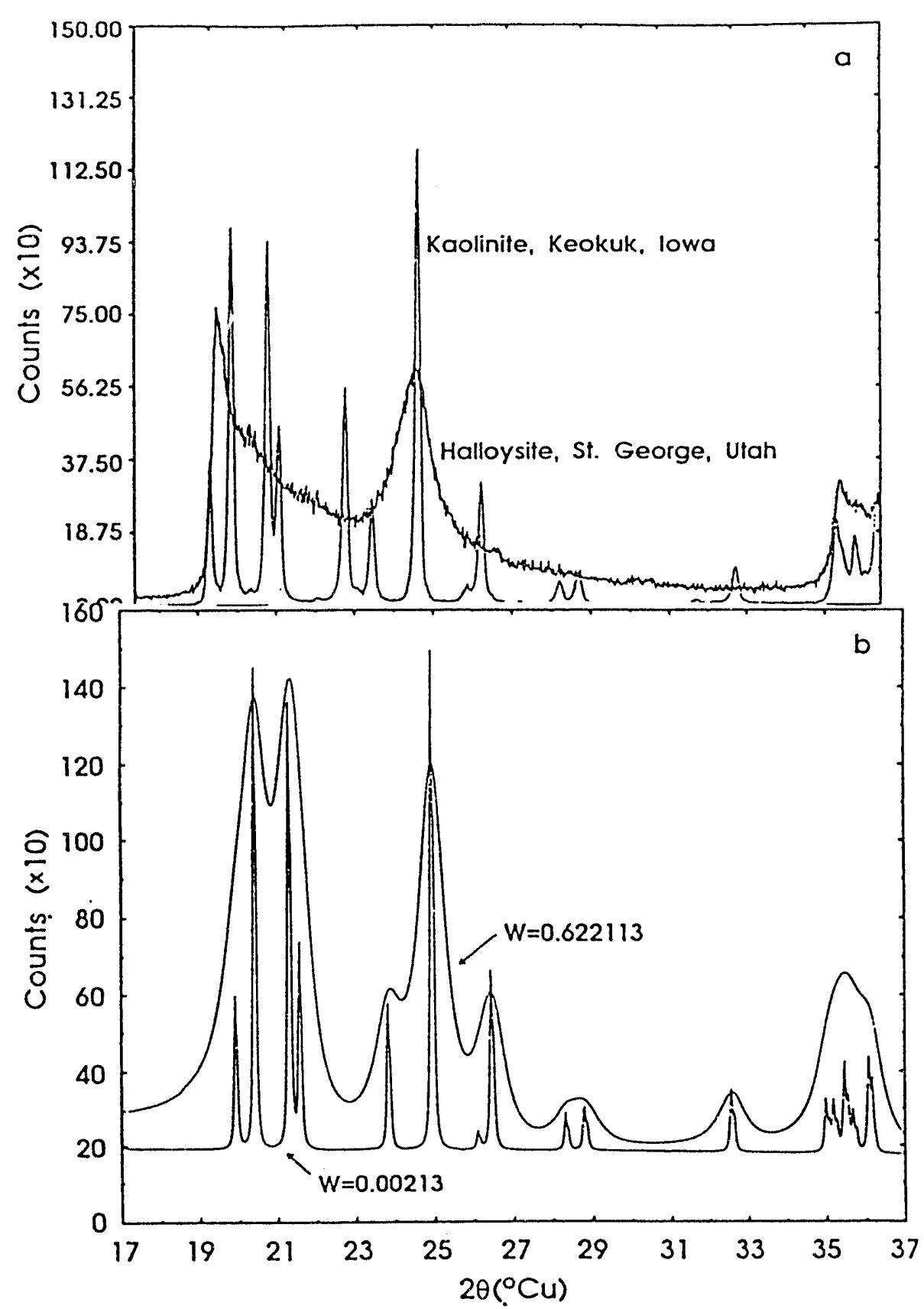

Figure 2. a) Observed pattems $(\mathrm{CuK} \alpha)$ of kaolinite and halloysite shewing the presence of $h k l$ reflections in the kaolinite pattern and two-dimensional diffraction bands in the halloysite pattem; and b) calculated kaolinite pattems broadened using two different $W$ values in the Caglioti et al. (1958) profile shape function. 

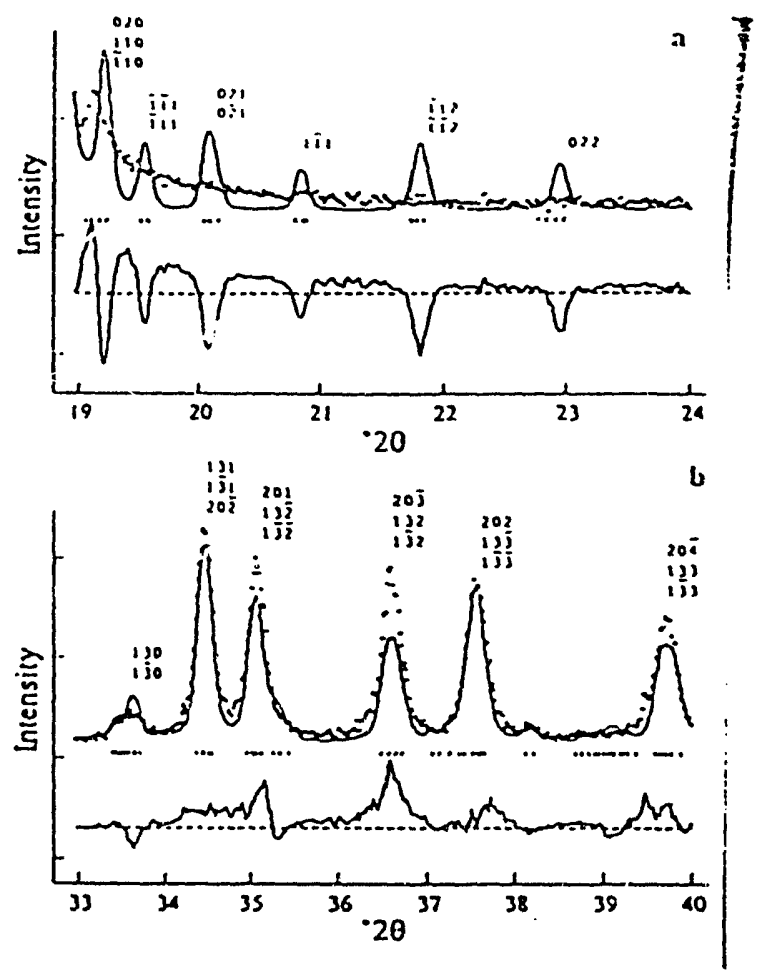

Figure 3. Portions of the observed (+) and calculated (solid line) diffraction patterns for a $\mathrm{Mg}$ chamosite; (a) data from 19 to $24^{\circ} 2 \theta^{2}$ (b) data from 33 to $40^{\circ} 2 \theta^{2}$. Solid curve at the bottom of each pattem represents the difference between observed and calculated patterns, and small -jid bars beneath observed data represent the calculated positions of all $\mathrm{CuK} \alpha 1$ and $\alpha 2$ refler.uons (from Walker and Bish, 1992).

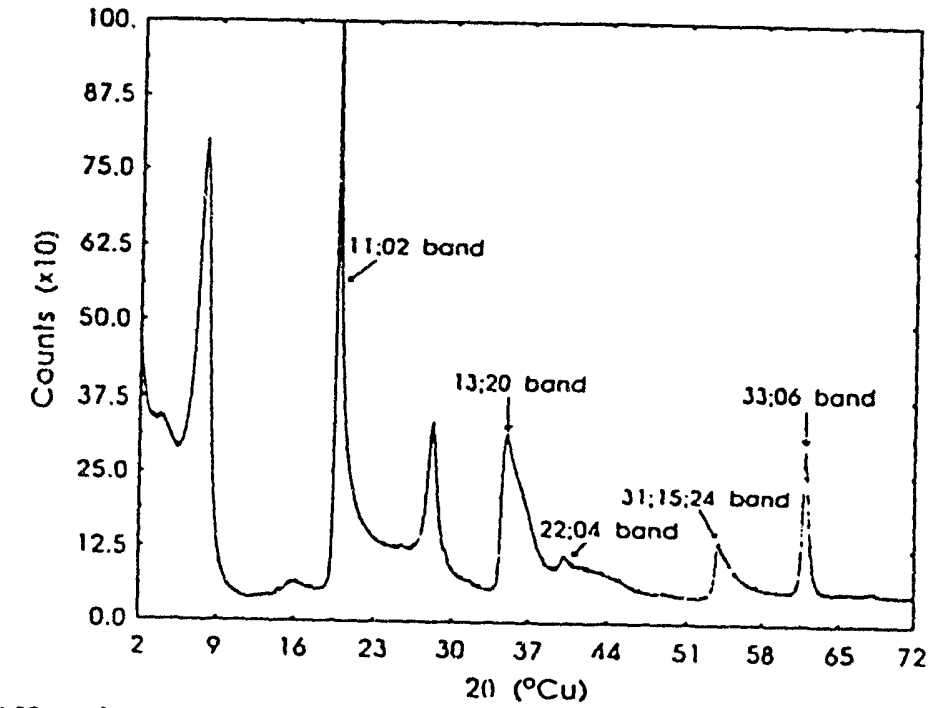

Figure 4. Observed diffraction pattem for a spray-dried Na-SWy-1 smectite. Note the sequence of $00 l$ reflections and the pronounced two-dimensional diffraction bands. 
Bish
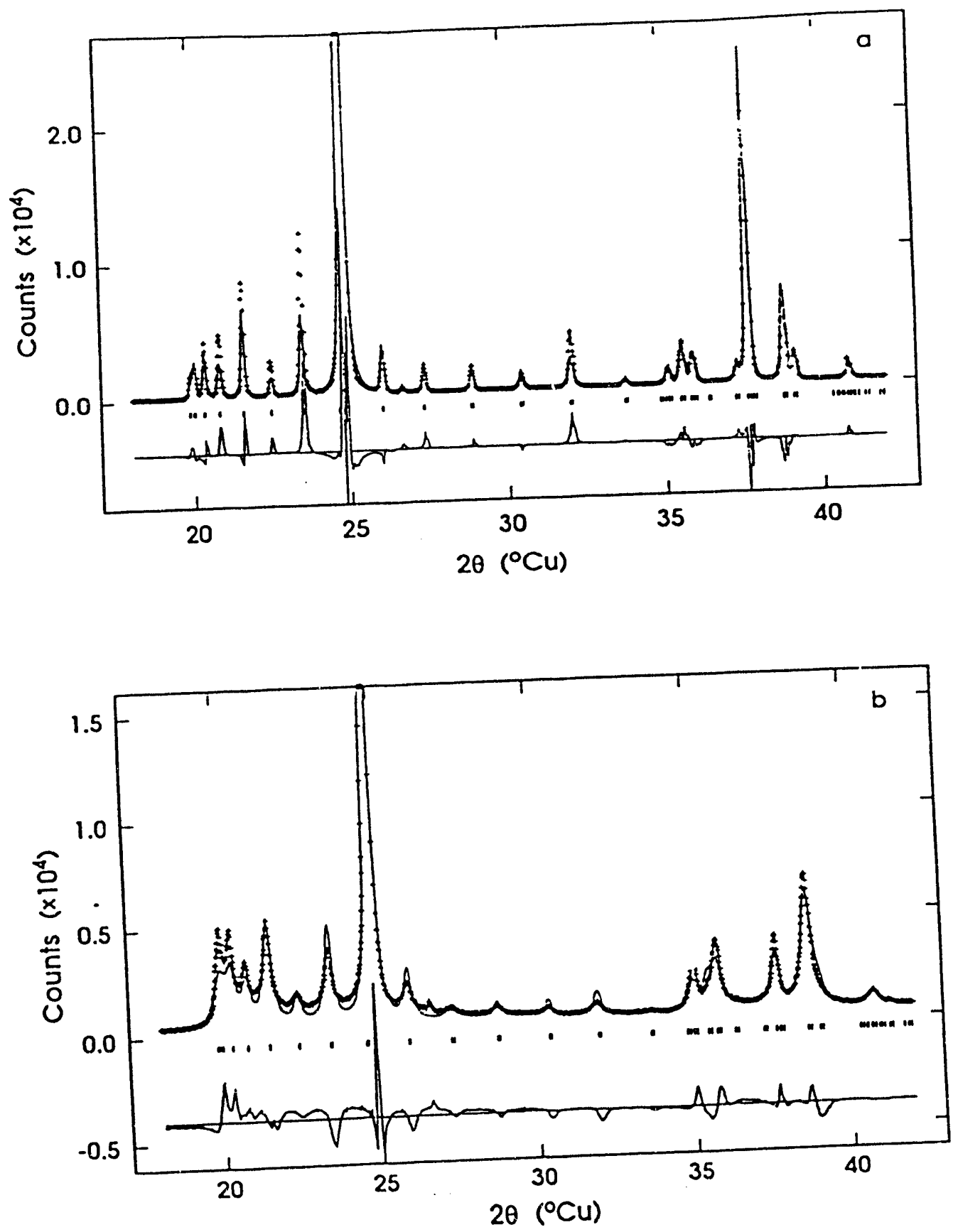

Figure 5. Observed (t) and calculated (solid-line) X-ray diffraction patterns (CuK $\alpha$ ) for a) unground dickite, in which the largest discrepancy is for the reflection just above $24^{\circ} 2 \theta$, due to preferred orientation of the dickite crystallites; and b) dickite that has been ground dry for one hour Note the enhanced "background" from $\sim 19$ to $28^{\circ} 2 \theta$, due to the presence of gris elevation in layer-stacking disorder, and the inability of the calculated $\mathrm{pat} \alpha \mathbf{1}$ and $\mathrm{K} \alpha 2$ reflections of dickite.

"background". Tic marks indicate positions for allowed Cukalated profiles.
Lower curve in cach is the difference between observed and calculated 
Studies of Clays Using the Reitveld Method

\section{APPLICATIONS OF THE RIETVELD METHOD TO CLAY MINERALS}

\section{Crystal Structure Refinements}

Dickite. The first three-dimensional refineme it of a clay mineral (dickite) was not published until 1981 (Adams and Hewat, 1981), although tie Rietveld method was first devised in 1967. Even then, the application was not well developed for use with X-ray powder diffraction data, so Adams and Hewat used constant-wavelength neutron powder diffraction data in their study. Using the dickite structure of Newnham (1961) (no hydrogen atoms) as a starting model, they were able to locate the hydrogen atoms using a combination of three-dimensional difference-Fourier syntheses and one-dimensional Fourier syntheses. The final Rietveld refinement, incorporating all four H's, yielded an arrangement for the non- $\mathrm{H}$ atoms no: significantly different from that determined by Newnham (1961). However, the $\mathrm{H}$ positions were significantly different from those determined by Giese and Datta (1973) using electrostatic modeling. These differences were ascribed to difficulties in accurately modeling $\mathrm{H}$ in the electrostatic calculations. However, Adams and Hewat perceptively commented that further advances in the application of the Rietveld method to the study of clays and clay minerals would require an improved technique for accommodating anisotropic broadening of reflections and some way of incorporating the types of disorder leading to twodimensional diffraction effects. As will be seen below, the former has been accomplished but the latter remains a major stumbling block.

Bish and Johnston (1993) re-examined dickite using low-temperature neutron powder diffraction data, the Rietveld method and difference-Fourier syntheses, and Fourier-transform infrared spectroscopy. Their refined non-hydrogen structure was essentially identical to other published structures, but their hydrogen positions were distinct. They were able to uniquely assign all four $\mathrm{O}-\mathrm{H}$ stretching vibrations in infrared spectra to individual $\mathrm{OH}$ groups in the dickite structure, and they provided a structural basis for the temperature dependence of all four O-H stretching vibrations. Contrary to published low-temperature infrared spectra, there is no evidence that dickite possesses symmetry lower than $C c$ at low temperatures.

Kaolinite. One of the first Rietveld refinements of a clay mineral using X-ray powder diffraction data was published by Suitch and Young (1983) who refined the structure of Keokuk, Iowa, kaolinite assuming space group $P 1$. Concurrently, Adams (1983) reported the results of a Rietveld refinement of St. Austell, Cornwall, kaolinite in space group $C l$ done with neutron powder diffraction data. These refinements have been discussed at length by Bish (1993).

The most recent X-ray Rietveld study of kaolinite was by Bish and Von Dreele (1989), who used $\mathrm{CuK} \alpha \mathrm{X}$-ray powder diffraction data to refine the non-hydrogen positions in the structure. Data were collected in two ranges, from $10^{\circ}$ to $90^{\circ} 2 \theta$ counting for 4.0 s every $0.02^{\circ} 2 \theta$ and from $80^{\circ}$ to $150^{\circ} 2 \theta$ counting for $12.0 \mathrm{~s}$ every $0.02^{\circ} 2 \theta$. This data-collection strategy is significantly different from that used in other Rietveld refinements in two ways. First, data are typically collected only to $\sim 100^{\circ} 2 \theta$ because of the incorrect impression that high-angle reflections are so overlapped that they are not useful. In addition, collecting data in multiple ranges provides highangle data with precision comparable to that for the more intense low-angle diffraction intensities. 
Bish and Von Dreele (1989) initially used the Suitch and Young (1983) results as a starting model. However, in spite of attempts to influence the refinement, including incorporating a large preferred orientation correction and using heavily-weighted soft distance constraints (the practice of including predicted interatomic distances as weighted observations, similar to a DLS refinement), their refinement converged to a result very similar to that obtained by Suitch and Young. It was only after using a DLS structure as a starting model that the refinement converged to a final structure with reasonable bond lengths and a significantly lower $R$ factor. These results strongly suggested that Suitch and Young (1983) and Young and Hewat (1988) obtained a false-minimuin structure for kaolinite. The refinement of Bish and Von Dreele (1989) also illustrated another benefit of Rietveld refinement. After refining the kaolinite structure using X-ray data for Keokuk kaolinite, systematic differences were seen between the observed and calculated data plots (Figure 6 ), particularly at $\sim 20.9,22.5$, and $23.5^{\circ} 2 \theta$ These differences were eventually attributed to the presence of $\sim 4 \%$ dickite that had been missed in the diffraction patterns by all previous investigators. Additional phases or errors in the model are very apparent because the Rietveld method fits a calculated pattern based on the model structure to the observed data.

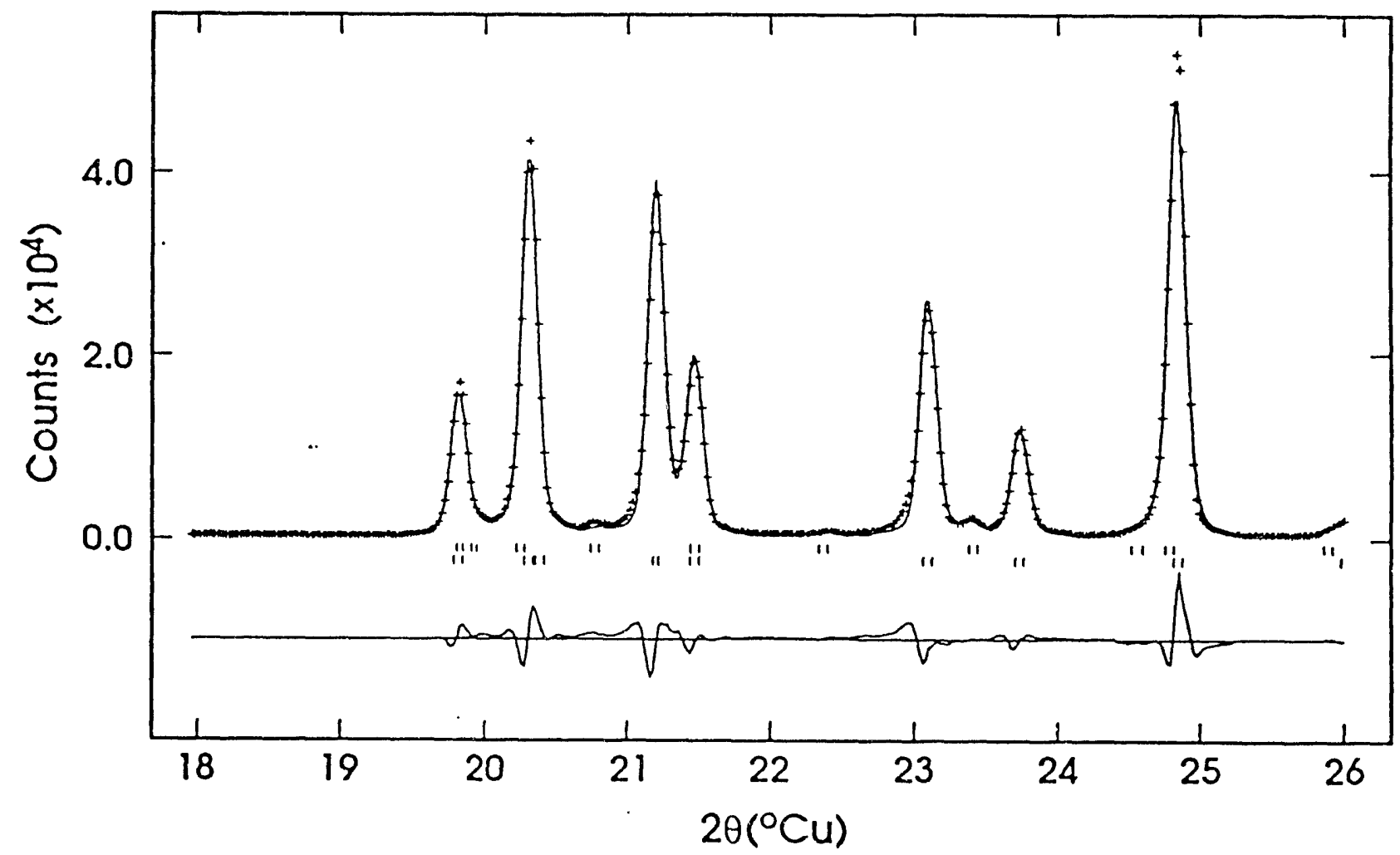

Figure 6. Observed (pluses) and calculated (solid-line) X-ray diffraction pattern for Keokuk kaolinite from $18.0^{\circ}$ to $26.0^{\circ} 2 \theta$, showing the presence of several dickite reflections. Tic marks indicate positions for allowed CuK $\alpha 1$ and $K \alpha 2$ reflecticns of dickite (upper) and kaolinite (lower). Small offset between observed and calculated reflection positions is due to a refined sampledisplacement correction, some of which is probably due to specimen transparency. Lower curve is the difference between observed and calcilated profiles (modified from Bish and Von Dreele, 1989). 
The low- and high-temperature structure refinements of Bish (1989) represent a funher application of the Rietveld method to kaolinite. He obtained X-ray powder diffraction data at 88 , 294 , and $573 \mathrm{~K}$ and performed complete structure refinements. From 88 to $573 \mathrm{~K}$, the $a$ and $b$ unit-cell parameters increased by only $0.05 \%$ and $0.01 \%$, respectively, whereas the $c$ parameter increased by $0.42 \%$. The total volume increase was $0.59 \%$. The markedly anisotropic thermal expansion is consistent with results obtained for other layer silicates. The atomic position results were of relatively low precision, but the cause of the anisotropic thermal expansion appeared to be a lengthening of octahedral $\mathrm{Al}-(\mathrm{O}, \mathrm{OH})$ bonds, resulting in a thickening of the octahedral sheet. These results are not in agreement with limited data for other layer silicates, in which the source of anisotropic expansion appears to be an increase in the interlayer separation. In fact, a recent lowtemperature Rietveld refinement of the kaolinite structure (Bish, 1994) confirms that the larger thermal expansion along the $Z$ direction is due to an increase in the interlayer separation.

Chlorite. Although many of the applications of the Rietveld method in clay mineralogy have been to minerals of the kaolin group, a few other clays and clay minerals have been studied using this method. Crystal structures of various chlorite polytypes have been determined primarily using single-crystal X-ray diffraction methods. Many chlorite samples exhibit random or semi-random stacking, and regular stacking sequences are relatively rare. Typical single-crystal studies of chlorites involve examination of many single crystals until a crystal with a regular stacking sequence is discovered (see Bailey, 1988, for a discussion of chlorite stacking sequences). Recently, Walker and Bish (1989), Rakovan and Guggenheim (1991), and Walker and Bish (1992) applied Rietveld refinement methods to several Il $b$ chlorite powders (samples filed, not ground), including two trioctahedral II $b$ chlorites (chamosite and clinochlore) and a II $b$ di, trioctahedral chlorite (sudoite). Both the chamosite and sudoite samples studied by Walker and Bish were somewhat disordered as judged by their $\mathrm{X}$-ray powder diffraction patterns. The chamosite refinement, in space group $C I$, yielded a final $R_{w p}$ of $15.7 \%$ and gave very precise unit-cell parameters. This $R_{w p}$ value is typical of those obtained with complex, poorly ordered materials; average $R_{w p}$ values for Rietveld refinements are larger and are not directly comparable to conventional $R$ values for single-crystal structure refinements. The final fit was good for $k=3 n$ reflections but was very poor for $k \neq 3 n$ reflections due to the presence of semi-random stacking in the sample (Figure 3). Although refinement of atomic positions for chamosite was not successful, site-occupancy refinements for the octahedral sites consistent with observed chemistry indicated that $\mathrm{Fe}$ and $\mathrm{Mg}$ were equally distributed between $\mathrm{M} 1, \mathrm{M} 2$, and $\mathrm{M} 3$, whereas $\mathrm{M} 4$ was occupied exclusively by Al. The March function preferred orientation correction for chamosite was 0.66 , which indicates a large degree of preferred orientation even though the sample was filed and back mounted. Although the final refined structure appeared reasonable because most of the atoms repeat at intervals of about $b / 3$, it was probably largely an artifact.

Results for the sudoite sample were obviously inferior to those for clinochlore even though the final $R_{w p}$ for sudoite was lower $(12.9 \%)$. Refinement of atomic positions was again unsuccessful, and site-occupancy refinements suggested the presence of $\propto$ ctahedral vacancies that were unsupported by compositional data. It thus appears that the occupancy data may be an artifact of the refinement. Preferred orientation was less for this sample (correction $=0.73$ ), due to the fine-grained narure of the powder. In spite of the difficulties in site-occupancy and atomic position refinements, precise unit-cell parameters were obtained, although they are of questionable accuracy" due to the lack of complete three-dimensional information. The clinochlore refinement of Rakovari 
Bish

and Guggenheim (1991) likewise exhibited evidence of systematic errors, atuributed by the authors to the presence of a two-layer II $b$ polytype intergrown with the dominant one-layer II $b$ polytype.

These chlorite refinements are good examples of the limits in applying Rietveld refinement methods to clay minerals. In spite of the fact that the refinements progressed normally and smoothly and yielded very precise unit-cell parameters and low $R_{w p}$ values, the refined structural information appears to be largely invalid. Problems with the refinements are most likely due to the presence of semi-random and random stacking sequences and to the existence of more than one polytype in the chlorite samples. Difficulties arise when attempting to determine structural information from non-Bragg reflections. However, even in the absence of regular stacking sequences, important information can be easily obtained from some chlorites, including precise unit-cell parameters and the relative distribution of heavy (e.g., $\mathrm{Fe}, \mathrm{Mn}, \mathrm{Ni}$ ) versus light (e.g., $\mathrm{Mg}, \mathrm{Al}$ ) atoms in octahedral sheets, although compositional data should be used to constrain the results of site-occupancy refinements. Better-ordered chlorites may yield reasonable and useful atomic positions if soft distance constraints consistent with known chemistry are used, although it appears probable that suitably ordered materials are very rare.

\section{Partial Structure Solution}

Difference-Fourier $(\Delta F)$ synthesis is a method used for refining crystal structures and is particularly well suited for locating scattering density missing from the structure model, such as interlayer species or hydrogen atoms in clay minerals. A $\Delta F$ synthesis is unaffected by series termination errors that occur when using limited amounts of data, and this method uses structure factors $\left(F_{\text {obs }}\right.$ ) obtained from observed intensities and those calculated using the model structure $\left(F_{\text {calc }}\right)$. It involves calculating the difference electron density function

$$
\Delta F(x, y, z)=\frac{1}{V} \sum_{h} \sum_{k} \sum_{l}\left(F_{o b s}^{h l d}-F_{c a l c}^{h e l}\right) \cos 2 \pi(h x+k y+l z)
$$

In this equation, $F_{\text {calc }}^{\text {kel }}$ represents the structure factors calculated using the assumed structure model and $F_{\text {obs }}^{\text {hth }}$ represents the observed structure factors with the same sign as $F_{\text {calc }}^{\text {htd }}$. There are a number of factors to consider when calculating $\Delta F$ maps, whether from powder or single-crystal diffraction data. First, in this calculation, the sign (or phase) of $F_{\text {obs }}$ is assumed to be the same as $F_{\text {cakc }}$. Therefore, if a significant amount of scattering density is missing or misplaced in the model structure, the calculated signs (phases) may be incorrect. For example, Rietveld refinements and $\Delta F$ calculations of synthetic $\mathrm{Cs} A$ and $\mathrm{Y}$ zeolites (Bish, unpublished) were fraught with difficulties because the omission of Cs atoms resulted in many incorrect structure factors (magnitudes and phases). Further, during the initial stages of refinement when some atoms are omitted, it is likely that temperature factors and scale factors will be incorrect, often by a large amount. An additional important consideration when calculating $\Delta F$ maps during Rietveld refinement is that the decomposition of overlapping peaks is performed assuming the ratios of the calculated (i.e., model-derived) intensities of the contributing reflections. Thus, results from a set of Rietveld refinements and $\Delta F$-map calculations may be very different from those that would be obtained from profile refinement, where observed intensities are generally model independent. In general, 
electron-density (or neutron-density) maxima are lower for Rietveld-derived $\Delta F$ maps than for those obtained from single-crystal data.

Location of missing atoms is always an iterative process because of these complications. Atoms are found, added to the model if reasonable (in scattering power and position), and another $\Delta F$ map is calculated after extraction of observed $F^{\prime}$ s. As more of the correct scattering material is added to the model, the calculated $F$ 's (magnitudes and phases) and scale factors become closer to the correct values, and the decomposition of overlapping peaks is improved. These complications also dictate how a Rietveld refinement must be performed in order to obtain reasonable calculated $F^{\prime}$ 's. Typically, few parameters are varied, and structural parameters are usually not refined. If structural parameters are varied, they may adjust in an attempt to compensate for the missing scattering power, yielding an unreasonable structure and complicating the interpretation of the $\Delta F$ map. In a Rietveld refinement $\Delta F$ map calculation, the scale factor(s) and background parameters are usually varied first, followed by the unit-cell parameters. Some profile parameters may be refined, although heavily overlapped patterns may require either fixing or manual adjustment of these parameters if observed and calculated patterns are significantly different. In practice, it appears that $\Delta F$ maps are relatively insensitive to a preferred orientation correction, although it is preferable to use data unaffected by orientation.

Hydrogen Atoms in Kaolinite. One of the more interesting aspects of structural studies of layer silicates and clay minerals is the location of the $\mathrm{H}$ atoms that so significantly affect their properies. The structure of kaolinite, $\mathrm{Al}_{2} \mathrm{Si}_{2} \mathrm{O}_{5}(\mathrm{OH})_{4}$, was outlined as early as 1930 , but it was not until 1983 that determinations of the locations of the $\mathrm{H}$ positions were first made using neutron diffraction data (Adams, 1983; Suitch and Young, 1983). Previous to 1983, Giese and Datta (1973) and Giese (1982) had modeled $\mathrm{H}$ positions using electrostatic modeling methods. Both Adams (1983) and Suitch and Young (1983) used published structures as starting models. Adams determined the $\mathrm{H}$ positions using $\Delta F$ maps, but his results appear to have been affected by disorder and two-dimensional diffraction effects in the sample. Suitch and Young assumed $\mathrm{H}$ positions for their starting model and they assumed a lower space group symmetry $(P I)$ than accepted for kaolinite $(C I)$, resulting in an apparent false-minimum structure. More recently, Young and Hewat (1988) re-refined the kaolinite structure with new neutron powder diffraction data, assuming a complete structural starting model and the lower space group symmetry; they obtained a structure similar to that obtained by Suitch and Young.

In 1990, Bish and Von Dreele re-examined the kaolinite structure using constant-wavelength neutron powder diffraction data and the non-hydrogen structure of Bish and Von Dreele (1989) as a starting model to reconcile the differences between the earlier refinements. It is important that no prior assumptions were made concerning the positions of $\mathrm{H}$ in kaolinite. They calculated $\Delta F$ maps, and the four largest negative re! ions of neutron density appeared reasonable for the four $\mathrm{H}$ positions (Figure 7). They then refined the complete structure, using $\Delta F$ maps and anisotropic refinements to examine the nature of the $\mathrm{H}$ sites. There was no significant anisotropy in any of the four $\mathrm{H}$ positions, although the inner-hydroxyl $\mathrm{H}$ appeared to be slightly smeared along the $z$ direction and the three inner-surface hydroxyl $\mathrm{H}$ atoms had larger displacement parameters within the plane of the layers. These results do not agree with those of Adams (1983), Suitch and Young (1983), or Young and Hewat (1988), and they do not supfort an ordered configuration of the $\mathrm{H}$ 


\section{Bish}

atoms that would require lowering the space group symmetry to $P_{i}$, as suggested by Suitch and Young (1983) and Young and Hewat (1988). It is noteworthy that Guthrie and Bish (1991) reproduced the refined $\mathrm{H}$ positions using an electrostatic minimization procedure. Calculated energy contours also showed that the inner-surface hydroxyl $\mathrm{H}$ atoms are situated in disk-shaped minima approximately parallel to (001) and the inner-hydroxyl $\mathrm{H}$ minimum is approximately perpendicular to $(001)$, in agreement with the results of the Rietveld refinement.

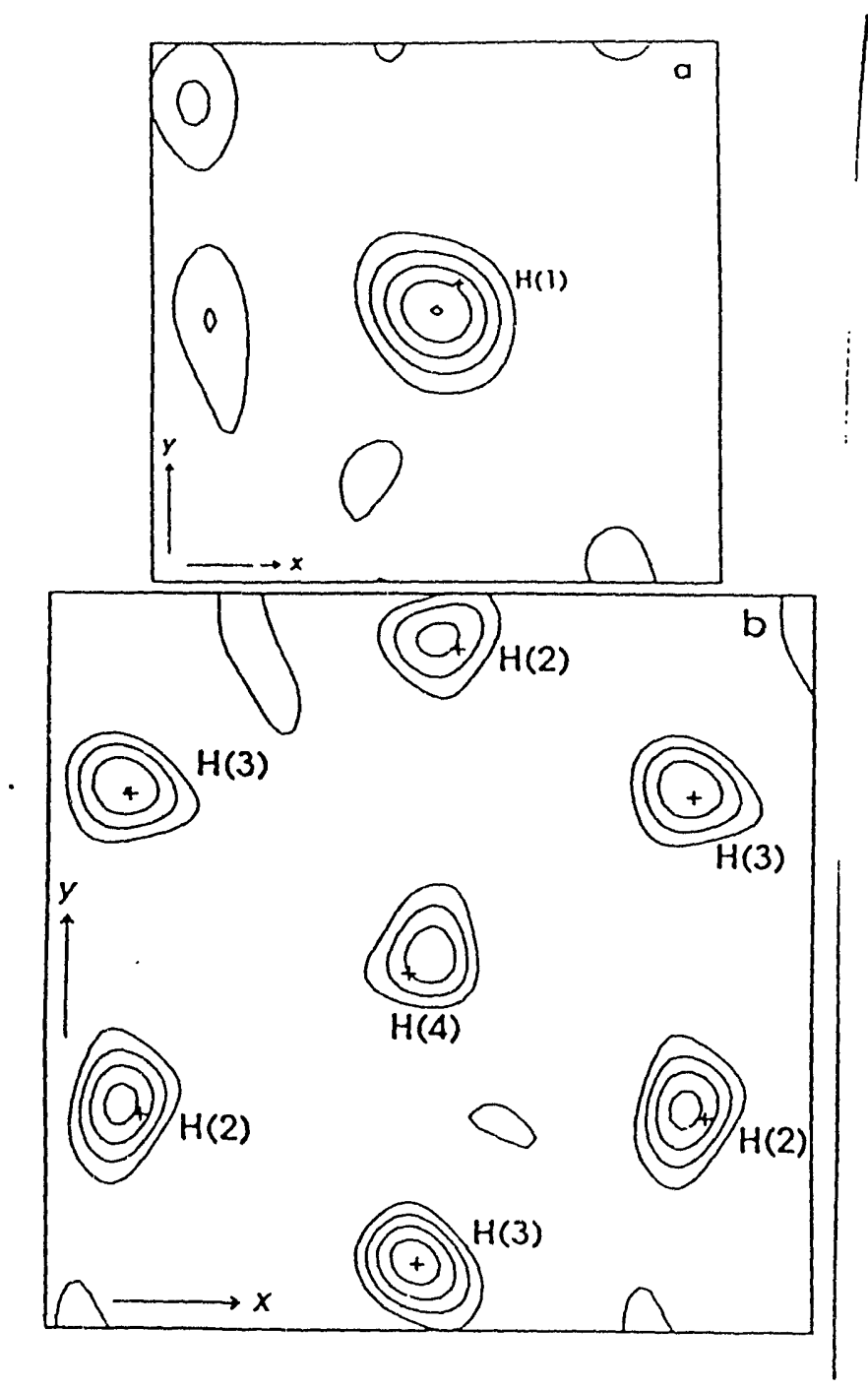

Figurc 7. Difference-Fourier maps obtained using neutron powder diffraction data for kaolinite a) in the region of the inner-OH atom, $z=0.293, x$ horizontal, $y$ vertical; $b$ ) in the interlayer region at $z=0.751, x$ horizontal, $y$ vertical. Conlours are drawn at $-0.25,-0.20,-0.15,-0.10$, and -0.05 for both maps, and plus $(+)$-ymbols represent the final refined positions for each $\mathrm{H}$ atom. 
Hydrogen Atoms in Dickite. Until recently, there was a similar lack of information on $\mathrm{H}$ positions in dickite, a polymorph of $\mathrm{Al}_{2} \mathrm{Si}_{2} \mathrm{O}_{5}(\mathrm{OH})_{4}$. In probably the first application of the Rietveld method to a layer silicate. Adams and Hewat (1981' examined the dickite structure using Fourier methods and neutron powder diffraction data. Their $\mathrm{H}$ positions, obtained on a sample exhibiting some two-dimensional diffraction effects, do not agree well with subsequent singlecrystal X-ray determinations (Sen Gupta et al., 1984; Joswig and Drits, 1986). Therefore, Bish and Johnston (1993) re-investigated the structure of dickite using low-temperature time-of-flight neutron powder diffraction data, the Rietveld method, and $\Delta F$ syntheses, in addition to variabletemperature Fourier-transform infrared (FTIR) spectroscopy. Using the dickite structure (obtained using single-crystal X-ray diffraction methods) of Joswig and Drits (1986) as a starting model, with no $\mathrm{H}$ atoms included, the four $\mathrm{H}$ atoms were unambiguously located as the four largest negative regions of density on $\Delta F$ maps. Figure 8 shows the three inner-surface hydroxyl $H$ atoms. The minor anisotropy noted for the $\mathrm{H}$ atoms in kaolinite was not observed for dickite. The observed $\mathrm{H}$ positions were most similar to the refinement of Joswig and Drits (1986), although the $\mathrm{O}-\mathrm{H}$ bond distances found by Bish and Johnston appear to be more accurate. The OH geometries, and the changes occurring from room temperature to $12 \mathrm{~K}$, are consistent with the FTIR spectra as a function of temperature and lend credence to the Rietveld-refined results. The relative ease with which the $\mathrm{H}$ positions were obtained in a powdered clay mineral such as dickite is remarkable when one considers that barely twenty-five years ago such information would have been out of reach using powdered samples.

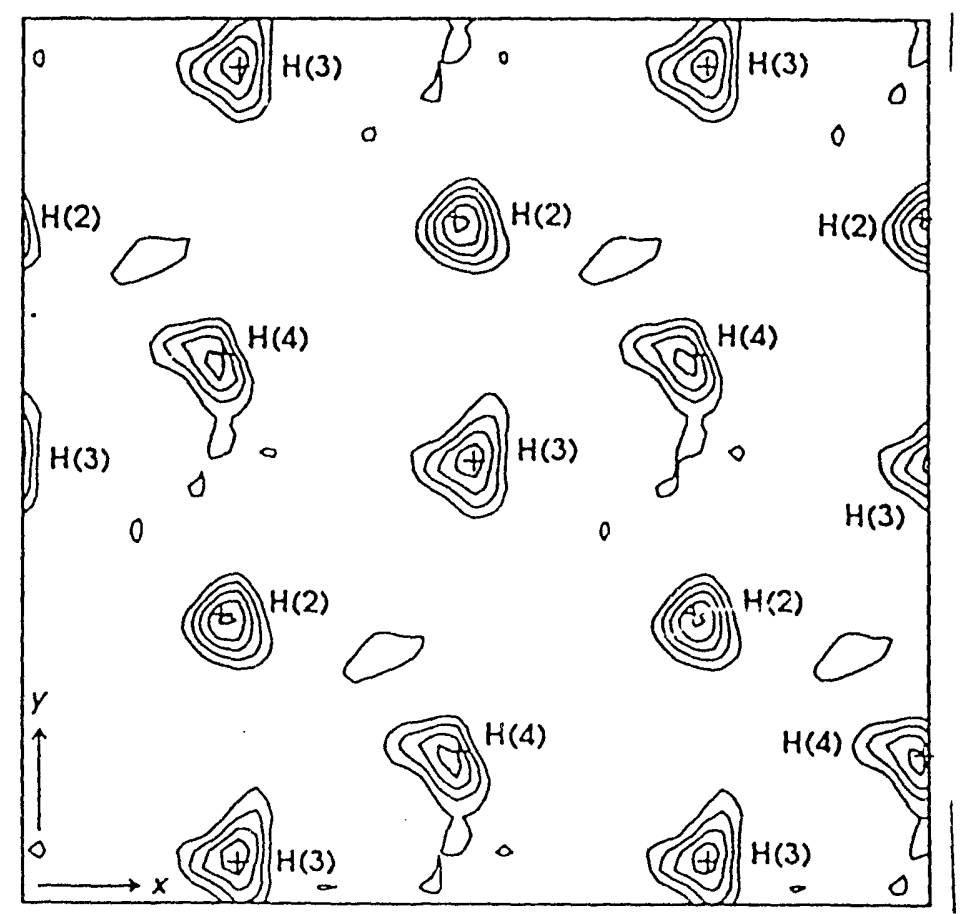

Figure 8. Difference-Fourier map for dickite in the interlayer region $(z=0.352, x$ horizontal, $y$ vertical) showing the positions of the inner-surface hydrogens. Contours are drawn at $-0.5,-0.4$, $-0.3,-0.2$, and -0.1 , and plus $(+)$ symbols represent the final refined positions for each hydrogen atom. 


\section{Bish}

It is important to make one final comment regarding the use of $\Delta F$ methods to locate $\mathrm{H}$ using neutron powder diffraction data. It is common practice in such experiments to attempt to replace $\mathrm{H}$ with $\mathrm{D}$ to reduce incoherent scattering. If the replacement can be made to go to completion, it should facilitate improved analysis due to a lowered background and improved signal-to-noise ratio. However, one can easily envision a situation in which only partial substitution of $D$ for $H$ is accomplished on a statistical basis. Such a result could produce $\Delta F$ maps revealing little or no information about the positions of $\mathrm{H}$ or $\mathrm{D}$ due to averaging of the negative neutron scattering length of $\mathrm{H}$ and the positive neutron scattering length of $\mathrm{D}$. One could create a material with an average neutron scattering length of zero in the H sites.

Interlayer Structure of Kaolinite Intercalates. The determination of the interlayer structure of the kaolinite-hydrazine intercalate is a good example of the utility of combined DLS modeling, $\Delta F$ methods, and Rietveld refinement Although kaolinite does not normally expand, it interacts strongly with several liquids to create expanded intercalates. When kaolinite is exposed to anhydrous hydrazine, $\mathrm{N}_{2} \mathrm{H}_{4}$, it expands to a material with either a 9.5- or a $10.4-\AA$ basal spacing, depending on the vapor pressure of water. Although the starting kaolinite may not possess good three-dimensional order, the $9.5-\AA$ complex often exhibits three-dimensional order sufficient to apply the Rietveld method. As a first step in the study of the $9.5-\AA$ intercalate, the structure was modeled using DLS, assuming the structure and $a, b, \alpha$, and $\gamma$ unit-cell parameters of $7-\AA$, nonexpanded kaolinite, and $\beta=114^{\circ}$, yielding a value for $c$ of $10.5 \AA$ which was obtained from a measured $d(001)$ of $9.52 \AA$. The assumption implicit in the DLS model was that the stacking of layers in the intercalate was the same as in unexpanded kaolinite. The DLS structure was used as the starting model for Rietveld refinement with $\mathrm{X}$-ray powder diffraction data, with the $\mathrm{N}_{2} \mathrm{H}_{4}$ molecule omitted. The two largest peaks in a $\Delta F$ map were in the interlayer region and were $1.40 \AA$ apar, consistent with a $\mathrm{N}_{2} \mathrm{H}_{4}$ molecule. The molecule appears to be canted within the interlayer, and there is evidence from the $\Delta F$ maps for positional disorder of the $\mathrm{N}$ atom closest to the hydroxyl interlayer surface (Figure 9). Inclusion of these two $\mathrm{N}$ atoms in the structure model and continued isotropic refinement yielded an $R_{w p}$ of $25 \%$, compared with $36 \%$ without the $\mathrm{N}$ atoms, although the refinement is still preliminary. This structure determination/refinement resolved the question of the exact orientation of the $\mathrm{N}_{2} \mathrm{H}_{4}$ molecule within the kaolinite interlayer, and the refinement result suggests that the intercalate has the same layer stacking as kaolinite. Given the low atomic number of the $\mathrm{N}$ and the poor quality of the material, it is remarkable that such a result was obtained with $\mathrm{X}$-ray data.

Comparable results were obtained using this methodology with a similar kaolinite intercalate. Costanzo et al. (1980) created a stable 8.4- $\AA$ hydrate of kaolinite by expanding the material with dimethylsulfoxide and then reacting the expanded material in a solution containing $\mathrm{NH}_{4} \mathrm{~F}$, thereby partially or completely fluorinating the interlayer hydroxyl groups. The resultant material has an enlarged $c$ repeat and three-dimensional order sufficient to apply the Rietveld method. The starting structure model was obtained using DLS methods, and the location of interlayer water molecules was determined from Rietveld refinement, $\mathrm{X}$-ray powder diffraction data, and $\Delta F$ maps (Bish et al., 1992). The largest peak on the $\Delta F$ maps was midway between the laj'ers, with only iwo other minor peaks above zero density. Rietveld refinement, incorporating an $\mathrm{O}$ atom at the position of the largest peak, yielded an $\mathrm{H}_{2} \mathrm{O}$ occupancy of 0.75 , positioned almost exactly midway between the silicate layers. Subsequent $\Delta F$ maps revealed no significant areas of electron density. 


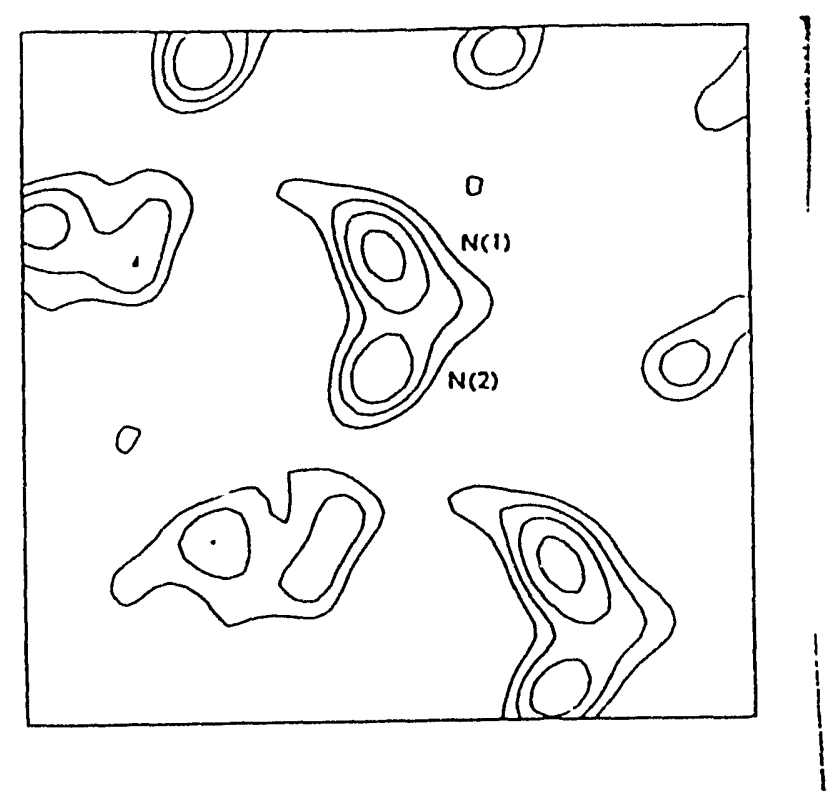

Figure 9. Difference-Fourier map for the kaolinite-hydrazine intercalate in the interlayer region $(z=$ $0.649)$, showing the positions of the two interlayer nitrogen atoms of the hydrazine molecule. Contours are drawn at $0.15,0.30,0.45$, and 0.65 .

Exchangeable Cations and Water in Sepiolite. Sepiolite would appear to be an ideal candidate for the use of Rietveld methods because it does not form large, well-ordered crystals. A schematic structure of sepiolite, $\mathrm{Mg}_{4} \mathrm{Si}_{6} \mathrm{O}_{15}(\mathrm{OH})_{2} \cdot 6 \mathrm{H}_{2} \mathrm{O}$, was proposed by Nagy and Bradley (1955) and Brauner and Preisinger (1956) using so-called fiber diagrams (two-dimensional data). However, the details of the atom distribution in the structural tunnels between the silicate chains remained poorly understood. In what constituted essentially the first three-dimensional structural study of sepiolite, Bish and Post (1987) studied a sample from Durango, Mexico, using a combination of Rietveld and difference-Fourier methods with X-ray powder diffraction data (Figure 10). Using the basic framework structure determined by Brauner and Preisinger (1956), they located and refined the positions of all water molecules postulatcd by Brauner and Preisinger, with the addition of two other water molecules. The "water of crystallization" site is fully occupied, whereas the remaining water sites (as judged by interatomic distances) are only partially occupied. Their results do not agree in detail with the electron diffraction results of Rautureau and Tchoubar (1974), and several of the split-atom positions proposed by Rautureau and Tchoubar were not confurmed. The greatest difficulty faced in interpreting the $\Delta F$ maps was deciding which peaks were real; all of the tunnel occupants are low atomic-number atoms, and most or all of the sites are probably partially occupied. In cases such as this, one must rely on crystal-chemical reasoning, using information such as bond distances and observed stoichiometry. Like the results of Adams and co-workers, these studies of sepiolite are examples of the utility of the combination of Rietveld and difference-Fourier methods for partial structure solution. Even partially occupied water sites in relatively poorly ordered materials can be located. 
Bish

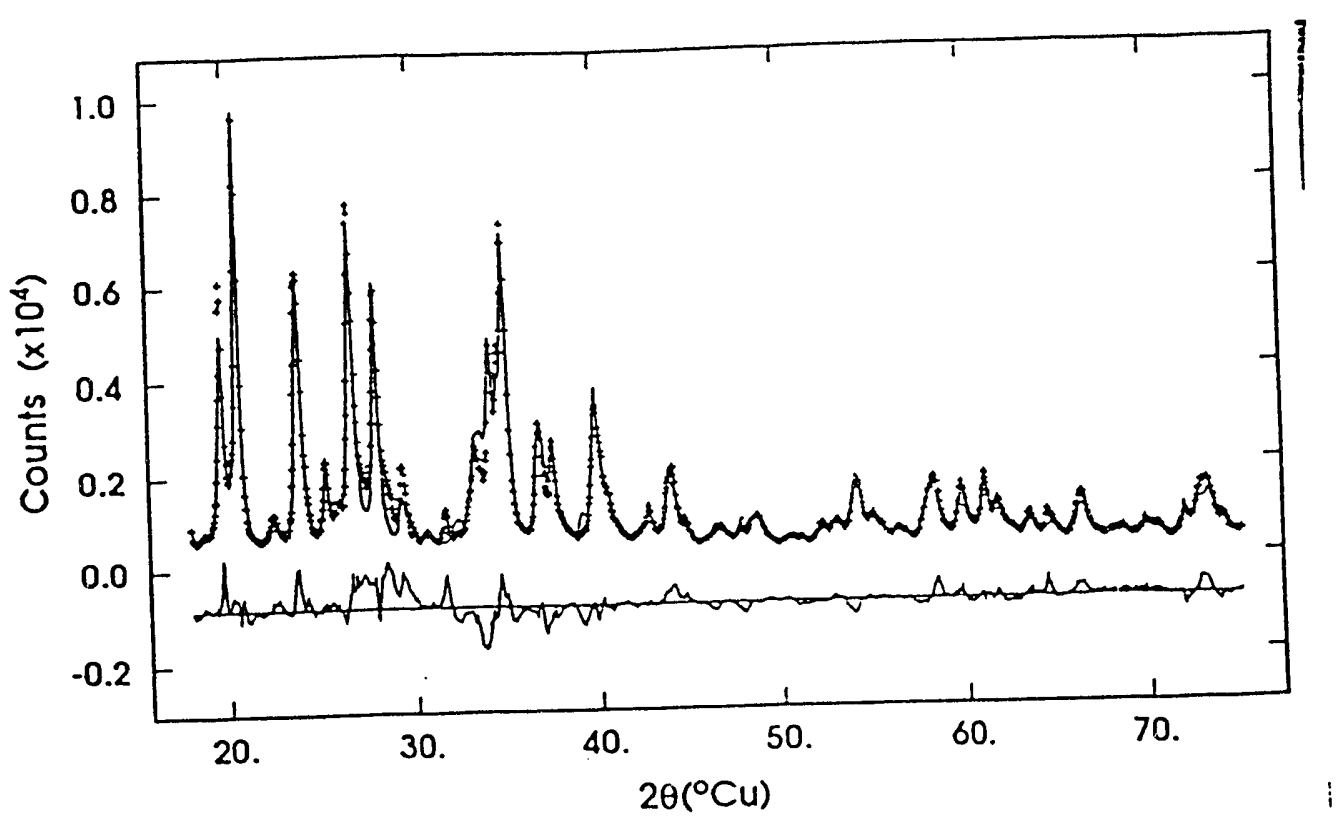

Figure 10. Observed $(+)$ and calculated (solid line) X-ray diffraction data (CuK $\alpha$ ) for sepiolite. Lower curve is the difference between observed and calculated profilcs.

\section{Quantitative Analysis}

Quantitative phase analysis using calculated patterns is a natural outgrowth of the Rietveld method because the refined scale factor for each crystalline phase is related to the amount of the material present in a mixture. The methodology involved in quantitative analysis using the Rietveld mechod is analogous to conventional single-phase Rietveld refinement, and the quantitative analysis theory is identical to that implemented in most conventional quantitative analyses (e.g., Klug and Alexander, 1974; Cullity, 1978).

Theory. The integrated intensity of $\mathrm{X}$-rays diffracted by a randomly oriented infinitely thick polycrystalline sample in flat-plate geometry utilizing a diffracted-beam monochromator can be written for a particular reflection as

$$
\begin{aligned}
I_{n t l}= & \left\{\frac{I_{0} A \lambda^{3}}{32 \pi r}\left(\frac{\mu_{0}}{4 \pi}\right)^{2}\left(\frac{e^{4}}{m^{2}}\right)\left(\frac{1}{2 \mu}\right)\left(\frac{1}{V^{2}}\right)\right. \\
& \left.x\left[|F|^{2} p \frac{1+\cos ^{2} 2 \theta \cos ^{2} 2 \theta_{m}}{\sin ^{2} \theta \cos \theta}\right] e^{-2 m}\right\}
\end{aligned}
$$


where the subscript $h k l$ denotes the dependence of particular terms on the B.r.gg reflection $h k l$. Here $I=$ integrated intensity per unit length of diffraction line (joules $\mathrm{sec}^{-1} \mathrm{~m}^{-1}$ ), $I_{\mathrm{O}}=$ intensity of incident beam (joules $\left.\mathrm{sec}^{-1} \mathrm{~m}^{-2}\right), A=$ cross-sectional area of incident beam $\left(\mathrm{m}^{2}\right), \lambda=$ wavelength of incident beam $(\mathrm{m}), r=$ radius of diffractometer circle $(\mathrm{m}), \mu_{0}=4 \pi \times 10^{-7} \mathrm{~m} \mathrm{~kg} \mathrm{C}-2, e=$ charge on electron $(\mathrm{C}), m=$ mass of electron $(\mathrm{kg}), V=$ volume of unit cell $\left(\mathrm{m}^{3}\right), F=$ structure factor, $p=$ multiplicity factor, $\theta=$ Bragg angle, $e^{-2 \mathrm{~m}}=$ temperature factor, and $\mu=$ linear absorption coefficient $\left(\mathrm{m}^{-1}\right)$, which enters as the absorption factor $1 / 2 \mu$ (Cullity, 1978). The term $2 \theta_{\mathrm{m}}$ refers to the diffraction angle of the diffracted-beam monochromator crystal. The constant $(K)$ and variable $\left(R_{h k l}\right)$ terms in (7) can be separated by defining

$$
K=\left(\frac{I_{0} A \lambda^{3}}{32 \pi r}\right)\left(\frac{\mu_{0}}{4 \pi}\right)^{2}\left(\frac{e^{4}}{m^{2}}\right)
$$

and

$$
R_{k t}=\left(\frac{1}{V^{2}}\right)\left[\mid F^{2} p\left(\frac{1+\cos ^{2} 2 \theta \cos ^{2} 2 \theta_{m}}{\sin ^{2} \theta \cos \theta}\right) e^{-2 m}\right]_{\omega} .
$$

Equation (7) can now be written in termr of (8) and (9) as

$$
I_{\text {hes }}=K(1 / 2 \mu) R_{\text {het }} \text {. }
$$

In a mixture, the intensity of the $h k l$ reflection from the $\alpha$ phase is given as

$$
I_{\alpha, \mu \omega}=C_{\alpha} K\left(1 / 2 \mu_{m}\right) R_{\alpha, h e l}
$$

where $C_{\alpha}$ is the volume fraction of the $\alpha$ phase and $\mu_{\mathrm{m}}$ is the linear absorption coefficient of the mixture. In terms of weight fraction ( $W_{\alpha}$, Eq. (11) can be rewritten as

$$
I_{\alpha, \text { hel }}=\left(\frac{W_{\alpha}}{\rho_{\alpha}}\right) K\left(\frac{\rho_{m}}{2 \mu_{m}}\right) R_{\alpha, \text { hed }} \text {, }
$$

where $\rho_{\alpha}$ is the density of phase $\alpha$ and $\rho_{m}$ is the density of the mixture. The Rietveld scale factor, $S$, in Eq. (2) includes all of the constant terms in Eq. (7) and for $X$-rays can be written

$$
S=\frac{K}{V^{2} \mu}
$$


where $V$ is the unit-cell volume and $\mu$ is the linear absorption coefficient for the sample. Thus, for a multi-phase mixture, Eq. (2) can be rewritten summing over the $p$ phases in a mixture (e.g., Hill and Howard, 1987) as

$$
y_{i}(c)=y_{i b}(c)+\sum_{p} S_{p} \sum_{k} p_{k p} L_{k p}\left|F_{k p}\right|^{2} G\left(\Delta \theta_{i b p}\right) P_{k p}
$$

The scale factor for each phase can now be written

$$
S_{\alpha}=\frac{C_{\alpha} K}{V_{\alpha}^{2} \mu_{m}}
$$

where $C_{\alpha}$ is the volume fraction of the $\alpha$ phase, $V_{\alpha}$ is the unit-cell volume of phase $\alpha$, and $\mu_{m}$ is the linear absorption coefficient of the mixture. Recasting Eq. (15) in terms of weight fractions and the mass absorption coefficient of the mixture we obtain

$$
S_{\alpha}=\frac{W_{\alpha} K}{\left(\rho_{\alpha} V_{\alpha}^{2} \mu^{*}\right)}
$$

where $\mu^{*}$ is the mass absorption coefficient of the sample, $W_{\alpha}$ is the weight fraction of phase $\alpha$, and $p_{\alpha}$ and $V_{\alpha}$ are the density and unit cell volume, respectively, of phase $\alpha$. Altematively, the unit-cell volume can be incorporated into the variable, phase-specific parameters as outlined by Bish and Howard (1988).

The scale factors contain the desired weight fraction information in a Rietveld analysis of a multicomponent mixture. However, the value of $K$ and the sample mass absorption coefficient cannot easily be determined so that an analysis of an unknown sample is usually performed by constraining to unity the sum of the weight fractions of the phases considered. Thus, for a twophase mixture,

$$
W_{\alpha}=\frac{W_{\alpha}}{\left(W_{\alpha}+W_{\beta}\right)}
$$

Equation (17) can be solved for the weight fractions of the $\alpha$ and $\beta$ phases to yield an expression for the weight fraction of phase $\alpha$ in terms of the scale factor information determined in the Rietveld analysis,

$$
W_{\alpha}=\frac{S_{\alpha} \rho_{\alpha} V_{\alpha}^{2}}{\left(S_{\alpha} \rho_{\alpha} V_{\alpha}^{2}+S_{\beta} \rho_{\beta} V_{\beta}^{2}\right)}
$$


In general, the weight fraction for the $i$ th component in a mixture of $n$ phases can be obtained from

$$
W_{i}=\frac{S_{i} \rho_{i} V_{i}^{2}}{\sum_{j} S_{j} \rho_{j} V_{j}^{2}}
$$

This method is exactly analogous to the adiabatic principle of Chung (1974b) in which reference intensity ratios are measured prior to analysis. The Rietveld method calculates absolute intensities to obtain intensities on an absolute scale rather than measuring reference intensity ratios.

A second method of Rietveld quantitative analysis requires that a known weight fraction of a crystalline internal standard be added to the unknown mixture. The internal standard can be any well-crystallized material that is readily available in pure form. Bish and Howard (1988) used Si, but corundum appears to be preferable (Linde $A$ corundum) (Bish and Post, 1993). If $W_{\alpha}$ is known, then an additional parameter $(C)$ can be evaluated from the internal standard:

$$
C=\frac{S_{\alpha} \rho_{\alpha} V_{\alpha}^{2}}{W_{\alpha}}=\frac{K}{\mu^{*}}
$$

This parameter can then be used to determine the weight fractions of other phases in the sample. For example, the weight fraction for the $\beta$ phase is determined by

$$
W_{\beta}=\frac{S_{\beta} \rho_{\beta} V_{\beta}^{2}}{C},
$$

where $S_{\beta}$ is the refinable scale factor for the $\beta$ phase, $\rho_{\beta}$ is calculated from the composition and cell parameters of phase $\beta$, and $C$ is determined using an internal standard and Eq. (20). Therefore, the weight fraction of phase $\beta\left(W_{\beta}\right)$ can be easily determined. This second method does not constrain the sum of the weight fractions, as does the first method, and it is analogous to internal-standard methods cummonly used to perform quantitative analysis such as the matrixflushing method of Chung (1974a). The total weight fraction of any amorphous components (or other phases neglected in the refinement) can thus be determined with this method if the amorphous profile can be Fit with the Rietveld background polynomial and if the amorphous content is significant (greater than 5-10\%). The difference between the sum of the weight fractions of the crystalline components and 1.0 is the total weight fraction of the amorphous components.

O'Connor and Raven (1988) used this method in slightly modified form. They determined the constant parameter, $K$, from a single sample and used the refined unit-cell parameters and cell contents to evaluate density and volume. The mass absorption coefficients were computed using the known compositions of the two phases in their single sample. It appears that some pitfalls may exist with this approach in light of their results on a 50:50 quartz:conndum mixture in which they concluded that their quartz contained $18 \%$ amorphous component. 
Bish

Application. Application of the Rietveld quantitative analysis method has been demonstrated on a variety of geological systems by Bish and Post (1993), but it is worth describing the application to mixtures containing clay minerals because significantly different problems arise. As emphasized above, the Rietveld method cannot be applied rigorously to materials exhibiting non-Bragg diffraction effects, such as many clays and layer silicates. With this caveat, the method can be successfully applied to a variety of important systems containing clays and clay minerals. Any materials in a mixture exhibiting non-Bragg diffraction effects can be analyzed by difference, using an internal standard. Alternatively, such materials can be included in a refinement in unusual cases. Guthrie and Bish (unpublished) successfully analyzed a synthetic mixture of dehydrated halloysite and corundum, assuming the dickite structure for halloysite. Only the scale factor and lattice parameters for halloysite were allowed to vary, and all other phase-specific parameters for halloysite were kept constant. The quantitative analysis was successful apparently because the useful observations for halloysite consisted only of $00 l$ reflections and the sample exhibited no preferred orientation of crystallites. The fit between observed $h k$ bands and calculated $h k l$ reflections was so poor that the bands did not appreciably affect the scale factor, i.e., there were signiticant errors above and below the calculated diffraction pattern. However, the poor fit between observed and calculated reflections produced a very large agreement factor. Such a situation may not always exist for samples containing clay minerals, so these mixtures should always be analyzed with caution.

The analyses of bauxite samples presented by Bish and Jones (1991) illustrate a successful application of the method to poorly crystalline soil samples. They analyzed a suite of Hawaiian bauxites taken from the surface to $290-\mathrm{cm}$ depth using $\operatorname{CoK} \alpha$ radiation. Although these samples represent complex (up to eight phases) and poorly crystalline mixtures, the refinements converged to consistent values and the resultant fits were excellent (Figure 11). The samples contained no materials exhibiting any two-dimensional diffraction effects so the Rietveld method could be applied without significant assumptions. The quantitative results were consistent with chemical data, and integrated peak intensities for the goethite 110 reflection found by profile fitting correlated very well with the quantitative results from Rietveld refinement $\left(r^{2}=0.96\right)$. Quantitative analyses for 12 samples are shown in Table 1 . In addition, the Rietveld refinements yielded precise unit-cell parameters for the major phases; for example, the goethite parameters suggested an Al content between 28 and 35\%, and the magnetite/maghemite parameters showed that they were $75-80 \%$ oxidized towards end-member maghemite (Table ?). The plots of X-ray diffraction data clearly showed that the goethite reflections were anisotrc?ically broadened, so refinements were conducted using the program GSAS (Larson and Von Dreele, 1988) that can explicitly model anisotropic crystallite-size and strain broadening. The results of the GSAS refinements suggested that the goethite reflections had a significant strain-broadening component in addition to a small crystallite size component. The crystallite-size broadening was primarily perpendicular to the (001) plane, whereas the strain broadening was principally in the $X-Y$ plane. The major phases in these samples did not exhibit significant preferred orientation, probably because of their fine-grained nature.

Interestingly, the Rietveld method can also be easily adapted to the analysis of trace phases. When the full pattern in Figure 12 was originally used in a multi-phase Rietveld quantitative analysis, refined amounts of erionite $(0.14 \%)$ were consistently above the known added amount of erionite $(0.05 \%)$. Further analysis of the "pure" analcime with the Rietveld method revealed the 
presence of $-0.07 \%$ erionite and $-0.43 \%$ illite, missed in the original analyses. The final Rietveld quantitative analyses are thus consistent with the amount of erionite in the analcime-erionite standard.

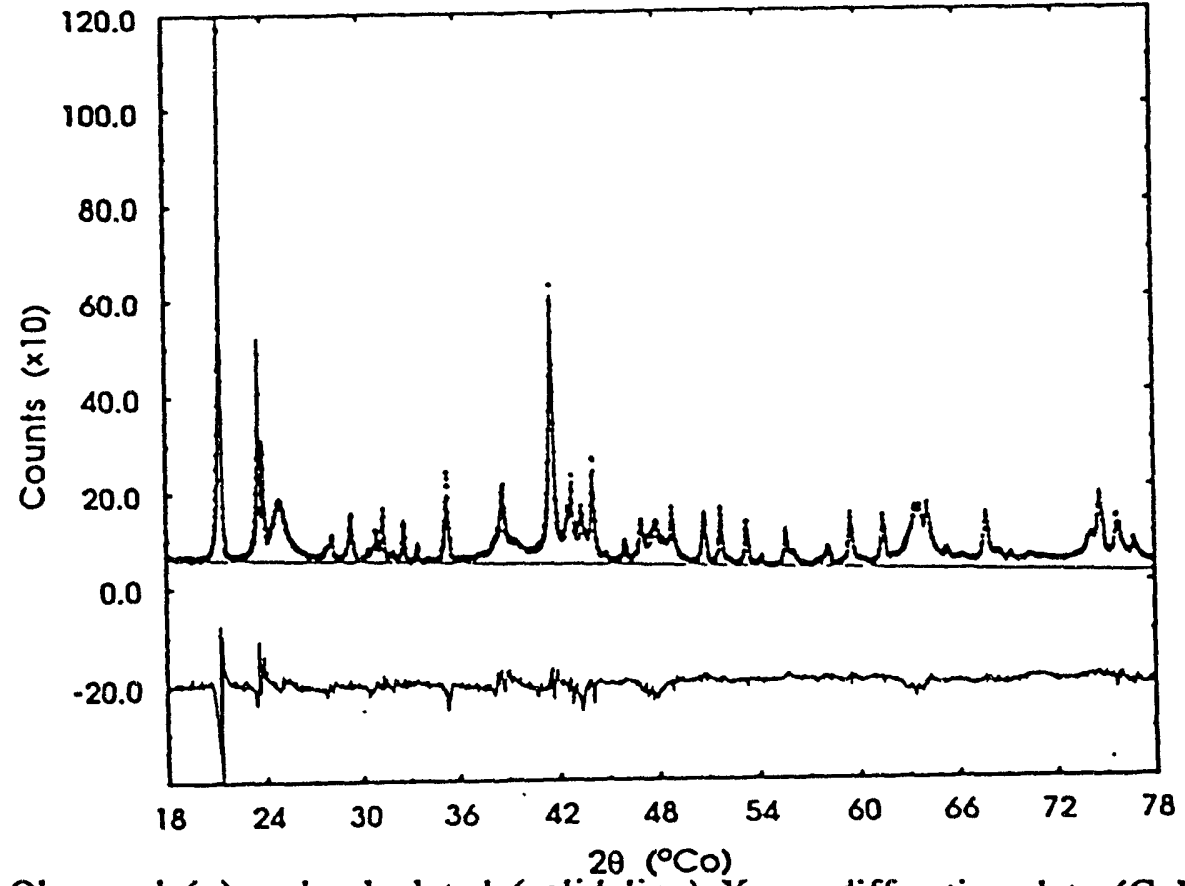

Figure 11. Observed ( + ) and calculated (solid line) X-ray diffraction data (CoK $\alpha$ ) for Hawaiian bauxite sample from 260- to $290-\mathrm{cm}$ depth. Lower curve is the difference between observed and calculated profiles.

Table 1. Quantitative analyses (wL.\%) of Hawaiian bauxites as a function of depth.

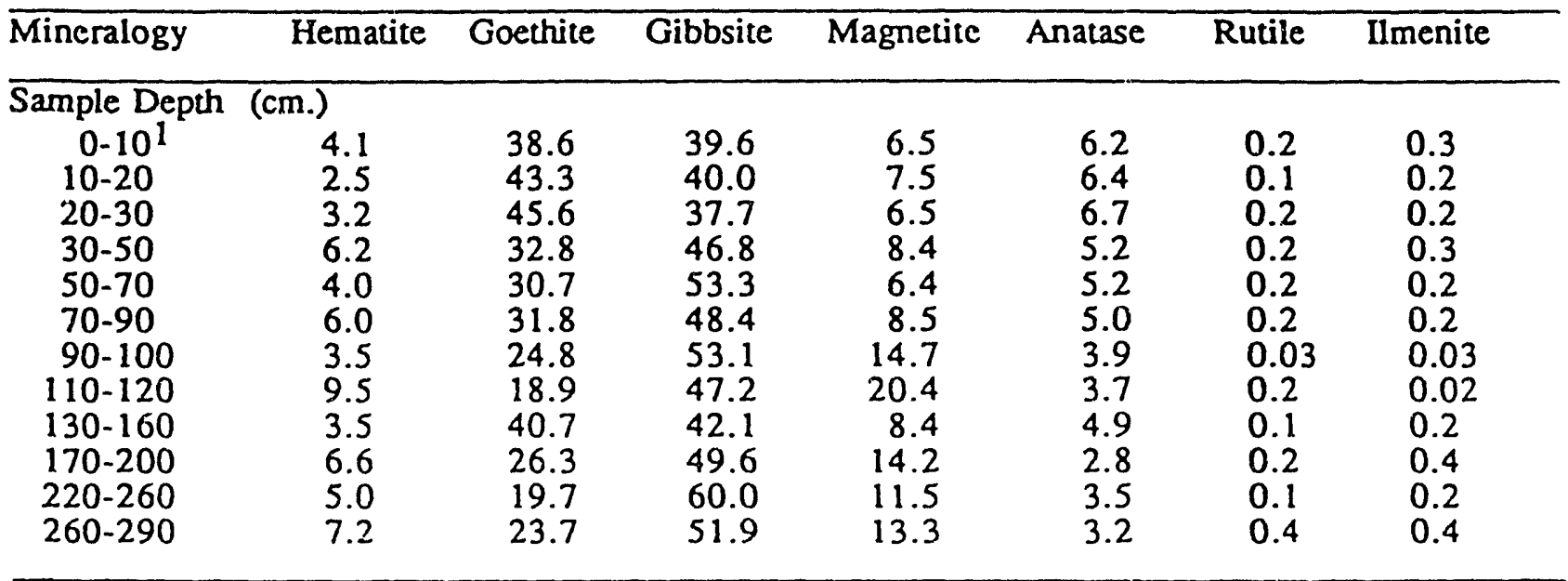

The $0-10 \mathrm{~cm}$ sample also contains $4.5 \%$ quarz which was not detected in all other samples 
Bish

Table 2. Unit-cell parameters $(\AA)$ for gocthitc and magnctite (maghemitc) for Hawaiian bauxite samples.

\begin{tabular}{lclll}
\hline & $a$ & $\begin{array}{c}\text { Gocthitc } \\
b\end{array}$ & $c$ & $\begin{array}{c}\text { Magnctitc } \\
a\end{array}$ \\
\hline Sample Depth (cm.) & & & & \\
& & & & \\
$0-10$ & $4.582(1)^{1}$ & $9.827(3)$ & $2.9731(6)$ & $8.3561(5)$ \\
$10-20$ & $4.584(1)$ & $9.843(3)$ & $2.9750(7)$ & $8.3588(8)$ \\
$20-30$ & $4.583(1)$ & $9.843(3)$ & $2.9758(7)$ & \\
$30-50$ & $4.587(2)$ & $9.849(5)$ & $2.973(1)$ & \\
$50-70$ & $4.584(2)$ & $9.855(5)$ & $2.972(1)$ & \\
$70-90$ & $4.587(2)$ & $9.864(5)$ & $2.976(1)$ & \\
$90-100$ & $4.580(2)$ & $9.812(7)$ & $2.969(2)$ & \\
$110-120$ & $4.595(4)$ & $9.84(1)$ & $2.964(2)$ & \\
$130-160$ & $4.581(2)$ & $9.841(5)$ & $2.9699(9)$ & \\
$170-200$ & $4.589(3)$ & $9.87(1)$ & $2.966(2)$ & \\
$220-260$ & $4.583(4)$ & $9.92(1)$ & $2.969(2)$ & \\
$260-290$ & $4.591(3)$ & $9.91(1)$ & $2.964(2)$ & \\
\hline
\end{tabular}

Numbers in paientheses represent estimated standard deviations in the last quoted decimal place.

\section{Refinement of Unit-Cell Parameters}

The Rietveld method minimizes the differences between observed and calculated diffraction patterns at every data point rather than relying on simpler methods that use only peak positions. In addition, calculated peak pcsitions are constrained by the model symmetry and by a set of unique unit-cell parameters, resulting in refined unit-cell parameters that are most consistent with the observed data. The calculated diffraction pattem also incorporates all possible reflections contributing to a peak that might shift its position slightly but may be too weak or too closely overlapped to be resolved. Therefore, the method yields unusually precise information on unit-cell parameters and reflection positions. Figure 13 illustrates a region of the diffraction pattern of clinoptilolite, showing the Rietveld fit to a composite three-peak region. The refinement results shown in the figure show that this three-peak region is instead composed of at least six reflections. Obviously, this overlap (common in powder diffsaction patterns) would make unique indexing of the peaks in the pattern difficult and would preclude accurate unit-cell parameter refinement by conventional methods.

The full-pattern fitting routine used in the Rietveld method also provides the opportunity to correct analytically for a number of the most serious systematic errors affecting peak positions, including specimen displacement, specimen transparency, and zero- $2 \theta$ offset. These effects, and corrections for them, are discussed by Klug and Alexander (1974) and Jenkins (1989). Incorporation of some or all of these effects allows the Rietveld method to provide unit-cell 
parameters not only of high precision but also of high accuracy. The Rietveld method appears to be superior to any other conventional method for determining unit-cell parameters.
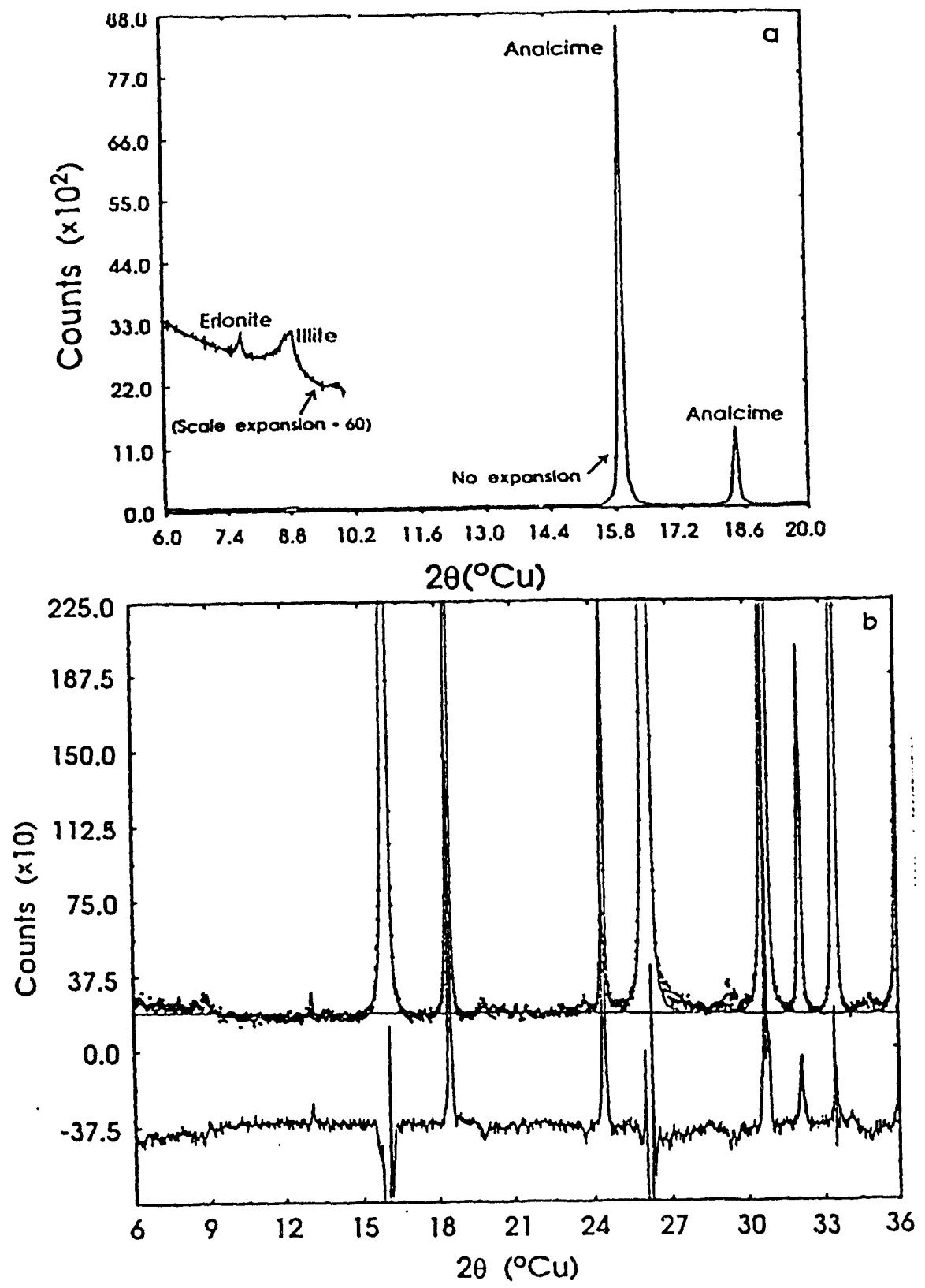

Figure 12. a) Observed X-ray powder diffraction pattem of a synthetic analcime-erionite mixture, showing the presence of $-0.14 \%$ erionite and $-0.43 \%$ illite; and b) observed $(t)$ and calculated (solid linc) $X$-ray diffraction data $(\mathrm{CuK} \alpha)$ for the analcime-erionite mixture. Lower curve is the difference between observed and calculated profiles. 


\section{Bish}

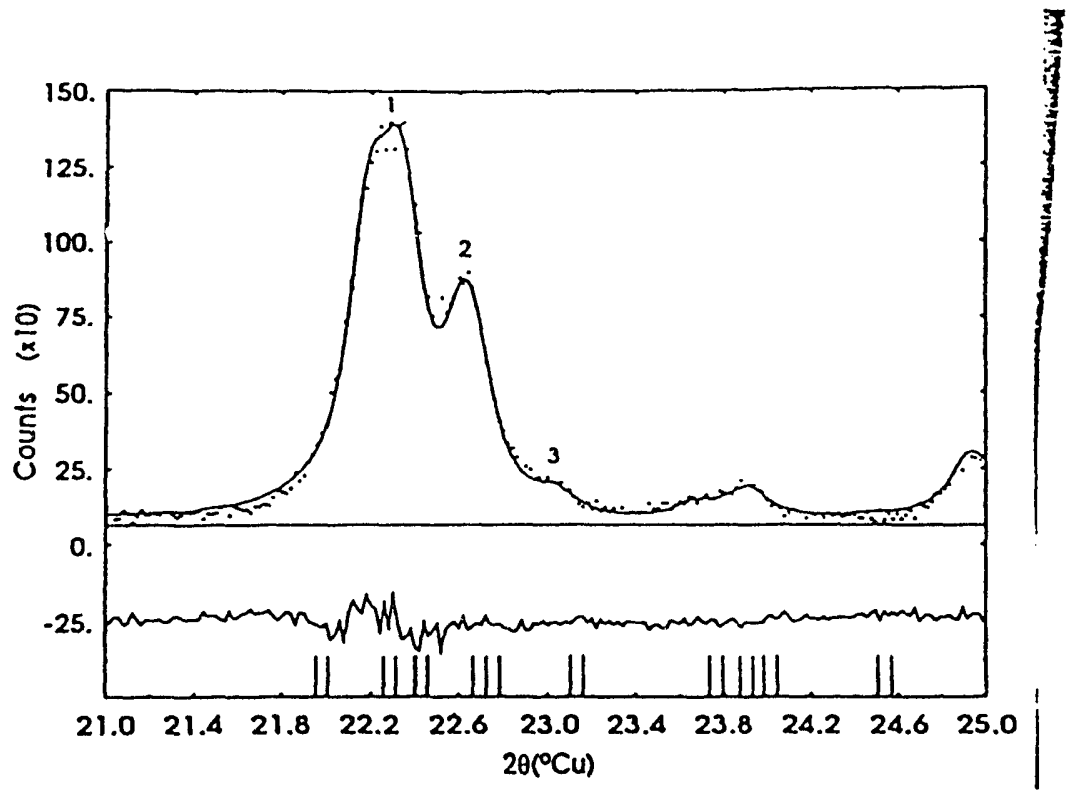

Figure 13. A portion of the observed ( + ) and calculated (solid line) powder $\mathrm{X}$-ray diffraction pattem of clinoptilolite, showing the fit to a three-peak (peaks are labeled) region. The lower curve shows the difference between observed and calculated patterns, and verucal marks (in pairs) at the bottom indicate the positions of allowed $\mathrm{K} \alpha 1$ and $\mathrm{Ka} 2$ reflections $\left(R_{w p}=14.7 \%, R_{\text {exp }}=\right.$ $7.5 \%)$.

Post and Bish (1989) illustrated that consistent unit-cell parameters could be obtained for $\mathrm{Si}$, even for samples displaced from the focal plane by up to 8 mils. They also gave refined unit-cell parameters for corundum, hematite, and quartz. Use of a specimen transparency correction in addition to a specimen displacement correction can yield even greater accuracy. Refinement of the unit-cell parameter of NIST $640 \mathrm{a}$ Si without either correction gave a value of $a=5.43073(2) \AA$ and use of both corrections gave a value of $5.43087(3) \AA$, comparing well with the certified value of $5.430825(36) \AA$ (statistically identical at the $1 \sigma$ level).

Application of the Rietveld method to synthetic Mn-goethite samples of varying $\mathrm{Mn}$ substitution (Bish and Ebinger, 1989) gave unit-cell values of $a=4.6056(4) \AA, b=9.9657(8) \AA$, and $c=3.0210(2) \AA$, significantly more precise than previous measurements. Precise values were obtained in spite of the presence of very broad reflections and a hematite impurity. It is difficult to assess the accuracy of these values because the true values are not known. The goethite $a$ and $c$ dimensions decreased by $0.039 \AA$ and $0.096 \AA$, respectively, and the $b$ dimension increased by $0.474 \AA$ as the $\mathrm{Mn}$ mole fraction increased to 0.47 . These changes in unit-cell parameters appear to be consistent with increasing Jahn-Teller distortion in the octahedral sites with increasing Mn substitution.

\section{Analysis of Peak Broadening}

Because the Rietveld method explicitly fits all observed diffraction peaks, considerable information on the profiles can be extracted, particularly if the capability exists to fit anisotropically 
broadened reflections. This treatment can potentially go far beyond the familiar Scherrer method which relates peak broadening to crystallite size. An analytical peak-shape function is explicitly included in all Rietveld refinements, a function that as accurately as possible represents the observed peak shapes. Although earlier Rietveld refinement programs used simple profile functions, such as a Gaussian or Cauchy profile, more recent programs use more complex functions such as a pseudo-Voigt (combination of Gaussian and Cauchy) or Pearson VII function (see Young and Wiles, 1982, for a description of many profile functions). These profile functions have commonly been combined with an angle-dependent peak-width function, the most common being the formulation of Caglioti et al. (1958) relating peak width, $H$, to angle:

$$
H=U \tan ^{2} \theta+V \tan \theta+W
$$

where $H$ is the peak full width at half-maximum height, and $U, V$, and $W$ are refinable parameters.

Instead of the Caglioti et al. (1958) formulation, the program GSAS (Larson and Von Dreele, 1988) uses a multi-term Simpson's-rule integration of the pseudo-Voigt function that contains separate expressions for Gaussian and Lorentzian broadening and incorporates the ability to accommodate anisotropic broadening. The Lorentzian component is parameterized in terms of $1 / \cos \theta$ and $\tan \theta$. As shown below, this formulation allows separation of crystallite size and strain contributions to reflection broadening.

Crystallite-size broadening causes all reflections in reciprocal space to be broadened alike, i.e., $\Delta d^{*}=$ constant. Because $d^{*}=d^{-1}, \Delta d^{*}=-d^{-2} \Delta d=\Delta d / d^{2}=$ constant, crystallite-size broadening is related to $2 \theta$ broadening by:

$$
\Delta d / d^{2}=\frac{\Delta \theta \cot \theta}{d}=\text { constant }
$$

Using Bragg's law, and the equality $2 \Delta \theta=\Delta 2 \theta$,

$$
\Delta d / d^{2}=\frac{\Delta 2 \theta \cot \theta \sin \theta}{\lambda} .
$$

Broadening is then:

$$
\Delta 2 \theta=\frac{\lambda\left(\Delta d / d^{2}\right)}{2 \cos \theta}
$$

Thus, crystallite size information can be extracted from a refinement if profile coefficients are parameterized to relate peak breadth to $1 / \cos \theta$. 
Bish

Strain (essentially a distribution of unit-cell parameters) broadening causes a shift in diffraction line position, and broadening in reciprocal space is a function of $d^{*}$, i.e. $\Delta d^{*} / d^{*}=$ constant. From the above relationships we can show therefore that $\Delta d / d=$ constant. Thus strain broadening in real space (as opposed to reciprocal space) is related to $2 \theta$ broadening by:

$$
\Delta d / d=\Delta 2 \theta \cot \theta=\text { constant }
$$

or

$$
\Delta 2 \theta=(\Delta d / d) \tan \theta(\Delta 2 \theta \text { in radians })
$$

Thus, if the profile coefficients are parameterized to relate peak breadth to $\tan \theta$, information on lattice strain can be extracted from a refinement. Broadening of a reflection (in $\Delta 2 \theta$ ) due to small crystallite size varies as the inverse of $\cos \theta$, whereas strain broadening (again in $\Delta 2 \theta$ ) varies as $\tan \theta$. Thus, determination of the pseudo-Voigt breadth terms related to $1 / \cos \theta$ and $\tan \theta \operatorname{can}$ provide information on crystallite-size and strain broadening, respectively.

Goethite. The Rietveld refinements of goethites (FeOOH) by Bish and Ebinger (1989) and Ebinger and Bish (1990) are good examples of the extraction of information on the sources of peak broadening in a mineral. Bish and Ebinger (1989) used these methods with X-ray powder diffraction data in a study of goethite and $\mathrm{Mn}$-goethites to determine not only structural information but insights into the causes of peak broadening for the samples (Figure 14). Their refinements illustrated that increasing Mn substitution in goethite causes a decrease in the $a$ and $c$ unit-cell parameters and an increase in the $b$ parameter. Atomic positions obtained for goethite were significantly different from limited literature data, and they showed that the environment of the metal site progressively changes as the amount of $\mathrm{Mn}$ substitution increases. The (Fe, $\mathrm{Mn})-\mathrm{O}, \mathrm{OH}$ distances systematically changed with an increase in $\mathrm{Mn}$ mole fraction. The two apical metal$\mathrm{O}, \mathrm{OH}$ distances lengthened significantly whereas the equatcrial metal-O,OH bonds shortened with an increase in Mn mole fraction, consistent with a Jahn-Teller distortion that increased with incorporation of $\mathrm{Mn}$. The relative changes in the unit-cell parameters are readily explained by these atomic changes. A new and interesting result of their study is the indication from the refined profile parameters that the principle cause of broad reflections in the $\mathrm{X}$-ray diffraction patterns is lattice strain rather than small crystallite size. These parameters implied that crystallite size either remained constant or increased with increasing $\mathrm{Mn}$ mole fraction. However, lattice strain increased with increasing $\mathrm{Mn}$, reaching values in excess of $5 \%$ strain at $0.47 \mathrm{Mn}$. Results also indicated that strain is greatest in the $X-Y$ plane. Bish and Ebinger concluded that the highly strained lattice is probably the reason for the observed structural break between goethite and groutite $(\mathrm{MnOOH})$. These results obtained from the observed profiles are in direct opposition to published results on goethite; all previous investigators had assumed the cause of broad reflections to be due to small crystallite sizes and had applied the Scherrer equation. Because of the extreme breadth of the goethite reflections and the presence of overlapping reflections (including contributions from hematite in the low-Mn materials) in the diffraction pattem, extraction of this information using other methods such as profile fitting would have been virtually impossible. 

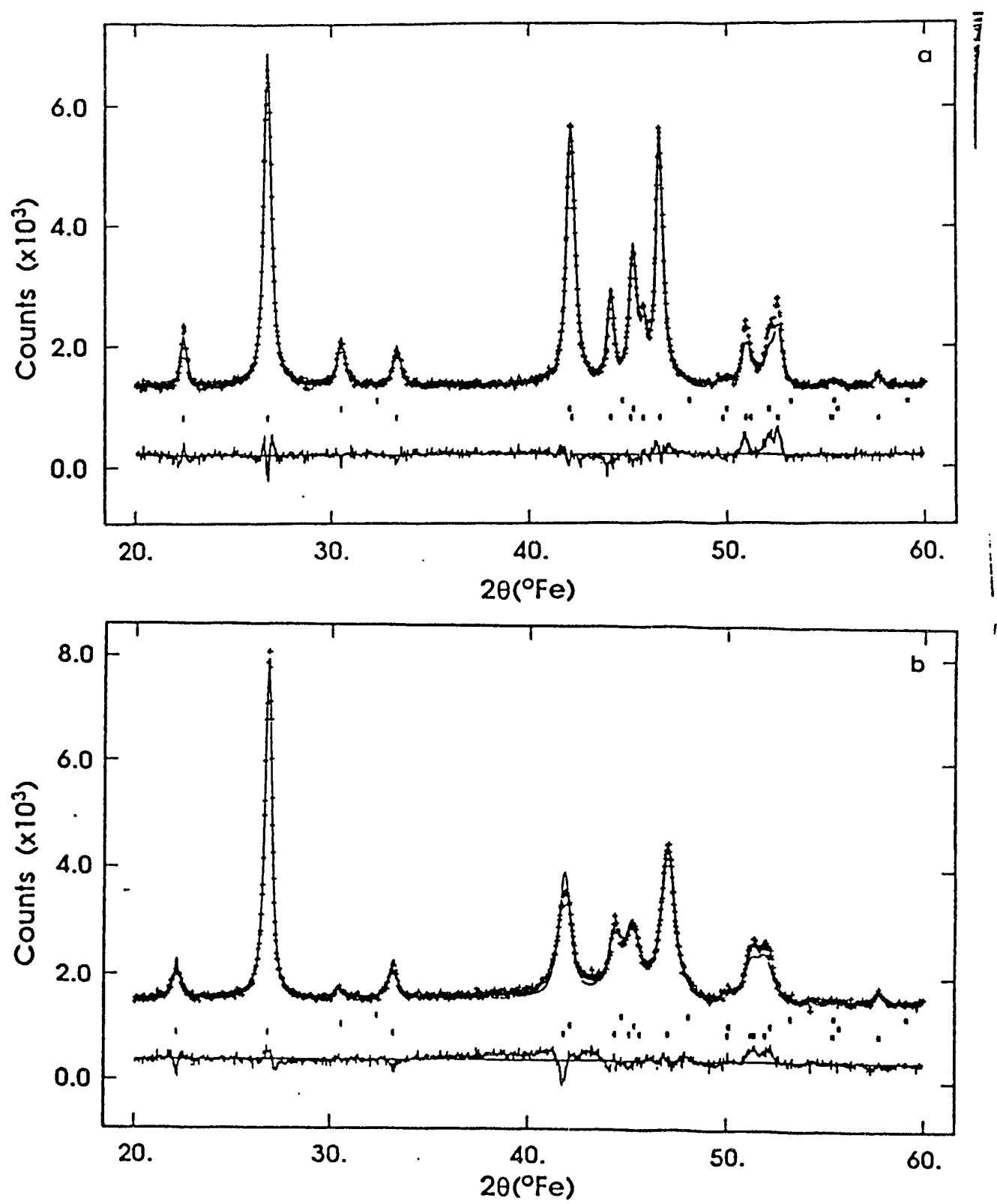

Figure 14. a) Observed $(t)$ and calculated (solid line) X-ray powder diffraction data (FeK $\alpha$ radiation) for synthetic goethite, FeOOH. Lower curve is the difference between observed and calculated profiles, and the tic marks indicate positions for allowed FeK $\alpha 1$ and $K \alpha 2$ reflections of hematite (upper) and goethite (lower). Goethite reflections are primarily crystallite-size broadened. b) Observed $(+)$ and calculated (solid line) X-ray powder diffraction data (FeK $\alpha$ radiation) for synthetic Mn-goethite, (Fe,Mn)OOH. Lower curve is the difference between observed and calculated profiles, and the tic marks indicate positions for allowed $F e K \alpha_{1}$ and $K \alpha_{2}$ reflections of hematite (upper) and goethite (lower). Gocthite reflections have a significant strainbroadening component. 
The Rietveld refinements of Ebinger and Bish (1990) on Cr-goethites are similar to those of Bish and Ebinger (1989) on Mn-goethites. The unit-cell parameters for $\mathrm{Cr}$-goethites decreased only a minor amount as $\mathrm{Cr}$ substitution increased, due to the similarity in size between $\mathrm{Cr}^{+3}$ and $\mathrm{Fe}^{+3}$. In contrast with the results for $\mathrm{Mn}$-goethites, the broadening of reflections in diffraction patterns for $\mathrm{Cr}$-goethites appeared to result primarily from small crystallite sizes rather than strain. This is consistent with the similarity in ionic size and the lack of Jahn-Teller distortions for $\mathrm{Cr}^{+3}$.

Clearly there are many potential applications of the Rietveld method to clay minerals in extracting information on the sources of peak broadening, because many clay minerals exhibit significantly broadened reflections. For example, the refinement of kaolinite by Bish and Von Dreele (1989) suggested that the kaolinite reflections were broadened by both crystallite size and strain effects, both of which were anisotropic. The strain broadening was greater along [001] than within the $X-Y$ plane, consistent with a finite distribution in the $c$ unit-cell parameter. Based on these results and those for goethite, it is probably fallacious to neglect the effects of strain broadening in studies of clay mineral diffraction peaks.

\section{SAMPLE REFINEMENT}

A typical Rietveld refinement is a complex undertaking, involving numerous variable parameters related to background, profile shapes, unit-cell parameters, preferred orientation, and structure. Due to the often large number of variable parameters, it is very easy to "get a pie in the face" by allowing parameters to vary too early in the refinement or allowing unjustified parameters to vary. In this respect, Rietveld refinements are very different from conventional single-crystal refinements in which individual reflection intensities or structure factors are used as observations. Because of the difficulties encountered during a Rietveld refinement, it is worthwhile to outline a typical and recommended refinement scheme that should minimize difficulties.

\section{Sample Preparation and Data Collection}

Perhaps the most important step in a Rietveld refinement is sample preparation and data collection as is the case with many instrumental methods of analysis. It helps to remember that a typical data collection may take as long as a day or two, unattended, whereas a typical Rietveld refinement may take much longer and require frequent user interventions. Thus, time spent on data collection is time well spent. It is always advantageous to use as pure a sample as possible, although most modem Rietveld programs incorporate the ability to treat multiple phases. Otherwise, the foremost requirement for a Rietveld refinement is accurate intensity data, so attempts must be made to obtain adequate particle statistics (using a sufficiently fine particle size) and to minimize preferred orientation. Bish and Reynolds (1989) suggested that particle sizes be at least as small as $10 \mu \mathrm{m}$ for materials of average linear absorption coefficient, and smaller if high linear absorption coefficient materials are used. As outlined by Bish and Reynolds, a variety of sample-mounting methods exist to reduce preferred orientation, but spray drying appears to produce the most "random" sample mount. If the Rietveld method is being used only for unit-cell parameter determination from a complex pattern, these requirements can be relaxed; relatively poor 
quality data can be used as long as no uncorrected systematic errors are present that can influence the reflection positions.

For both X-ray and neutron studies, it is recommended that data be collected out to the highangle limit of the instrument. This will provide much more stable refinement of angle-dependent variables such as temperature factors, site occupancies, and profile parameters (yielding information on crystallite size and strain broadening). Although it is a common perception that little or no useful information is available from the high-angle portion of diffraction patterns, this is usually not true. If a sample is sufficiently ordered and exhibits only Bragg diffraction effects, high-angle data will probably be useful. The high-angle data may appear broad and poorly resolved, but this is usually due to low intensities and overlapping of numerous reflections. The low-angle cutoff in data collection should be determined based on beam coverage of the sample and on instrumental effects. Low-angle reflections can have considerable asymmetry in both $X$-ray and fixed-wavelength neutron data, due in part to axial divergence of the beam (incident-beam Soller slits should be used). In addition, it is often worthwhile to avoid using data in the angular range in which Lorentz and polarization effects are rapidly varying. In any X-ray experiment, the sample must be fully within the incident beam at the lowest angle of interest, although a correction can be applied to the data if the $X$-ray beam and sample dimensions are known. Choosing a low-angle cutoff as high as $20^{\circ} 2 \theta$ seldom results in the loss of many reflections. Finally, it is important to note that a well-aligned diffractometer and well-known calibration standards are important in any Rietveld refinement. Although most Rietveld programs provide the ability to refine both zero-point and sample-displacement corrections, these two corrections are highly correlated and simultaneous refinement of both values may yield unstable results. Use of a diffractometer with no zero-point error and refinement of only a sample displacement correction is preferable.

It is possible and advantageous to obtain digital $X$-ray data in a variety of separate angular ranges in an attempt to acquire data with approximately constant precision. Consider a diffraction pattern in which reflections between 10 and $50^{\circ} 2 \theta$ are on the average five times as intense as those between 50 and $100^{\circ} 2 \theta$, which are in turn five times stronger on average than those between 100 and $160^{\circ} 2 \theta$. An appropriate data-collection strategy would then involve collection of three separate ranges, for example counting for $2 \mathrm{~s} / \mathrm{step}$ in the furst range, $10 \mathrm{~s} / \mathrm{step}$ in the second range, and $50 \mathrm{~s} / \mathrm{step}$ in the third range. The author has obtained good results using this data-collection strategy in conjunction with a Rietveld program that facilitates the use of multiple data sets (e.g., GSAS, Larson and Von Dreele, 1988). The use of multiple ranges is particularly beneficial for Xray diffraction studies because of the decrease in atomic scattering factors with angle. However, neutron scattering lengths do not decrease as a function of angle, so the multiple-range method is not necessary in neutron diffraction studies. The author has found that the use of this datacollection method with $\mathrm{X}$-ray diffraction data yields improved values for temperature factors and site occupancies, and individual temperature factors can be stably refined in favorable cases with complex structures. Post and Bish (1989) outlined the various arguments concerning choice of step sizes and count times for use in Rietveld data collection, and the interested reader should consult that discussion and the references cited therein. 
Bish

\section{Refinement Strategies}

To reiterate, the approximate crystal structures of all phases in a given sample must be known before performing a Rietveld refinement. The structures, together with the unit-cell parameters and space group symmetry of each phase, make up the sample-related parameters to be used as input to any Rietveld refinement program. In addition, before beginning a refinement, the types of background and profile functions to be used must be selected; the available options will be determined by the particular Rietveld program used. Most refinements with X-ray diffraction observations today use either pseudo-Voigt or Pearson VII profile functions, or a more complex variant of one of these. Appropriate starting values for the profile function are what are known as instrument parameters, i.e., values unaffected by any sample-related broadening. These values should be determined for an individual instrument with an appropriate material that contributes little or no sample-related broadening (e.g., NIST SRM 660, $\mathrm{LaB}_{6}$ ) so that instrument-related profile broadening can be evaluated and incorporated into the analysis of profiles. Table 3 illustrates a typical input file for a one-phase refinement of the structure of goethite for the DBW program of Wiles and Young (1981). This table is not meant to provide a template from which to prepare a Rietveld input file, but it is presented to illustrate the type of information typically required to perform a Rietveld refinement. As is obvious, there are numerous control parameters that define various instrumental, data, and refinement-related input values. Input for each phase consists of a title line, a line listing the number of atoms in the asymmetric unit, and, possibly, a preferred orientation direction. Following this information is a line containing the space group, and all atoms in the asymmetric unit are listed next. Input for each atom consists of one line providing the $x, y$, and $z$ atomic parameters, an isotropic temperature factor, and the site occupancy times the site multiplicity. The second line for each atom consists of six anisotropic temperature factor values, often set to zero in a Rietveld refinement. The next line contains the scale factor and an overall temperature factor, followed on the next line by the $U, V$, and $W$ parameters of Caglioti et al. (1958), a profile number (in this case Pearson VII), the value of the Pearson VII coefficient and its angle-dependence. Starting unit-cell parameters are contained on the next line, followed by a line containing information on a preferred-orientation correction and an asymmetry correction. The remainder of the file contains a variety of what are termed codewords that define which refinable parameters will be refined. Many varieties of input files exist for Rietveld refinement programs, and this table simply provides an example of the type of structural information and refinement parameters that are required.

Although the refinement strategy outlined here works well for the author, other similar strategies are used, and this procedure is by no means universally accepted in detail. After preparation of an input file containing the sample-related parameters and appropriate background and profile parameters, refinement can be commenced by allowing individual scale factors for each phase to vary. This stage of refinement is of ten combined with variation of background parameters (if fixed background points are not used). The aim at this point is to bring observed and calculated diffraction patterns to the same scale and to generate a background that closely matches the observed background. If a refined scale factor is too low, calculated intensities will be uniformly low by a constant factor, non-uniform intensity discrepancies can result from a variety of factors, including incorrect temperature factors, incorrect Lorentz-polarization factor, and errors in site occupancies or atomic positions. If the starting structural and unit-cell parameters are reasonably close to the true values, this first stage of refinement is usually successful. On some occasions, 
various parameters may require damping to remain stable, i.e., applying less than the full calculated shift to the parameter during each least-squares cycle. Throughout every stage of a refinement, it is important that progress be monitored by examining plots of observed and calculated data, particularly if any difficulties are encountered. Thus, it is imperative that quick plotting capabilities be available, e.g., on a graphics terminal. Any problems with the refinement, e.g., improper background parameters or scale factor, incorrect unit-cell parameters, incorrect profile parameters, or a missing phase, are usually readily apparent in the plots. It should also be obvious that a Rietveld refinement usually progresses in discrete steps, with refinement of a given set of parameters to convergence, addition of another parameter, and re-refinement to convergence. Virtually any variable may refine to unreasonable values (e.g., negative or very large temperature factors, negative profile parameters), and care should be exercised when adding additional refinable parameters.

Table 3. Sample goethite input file for the DBW Rietveld program.

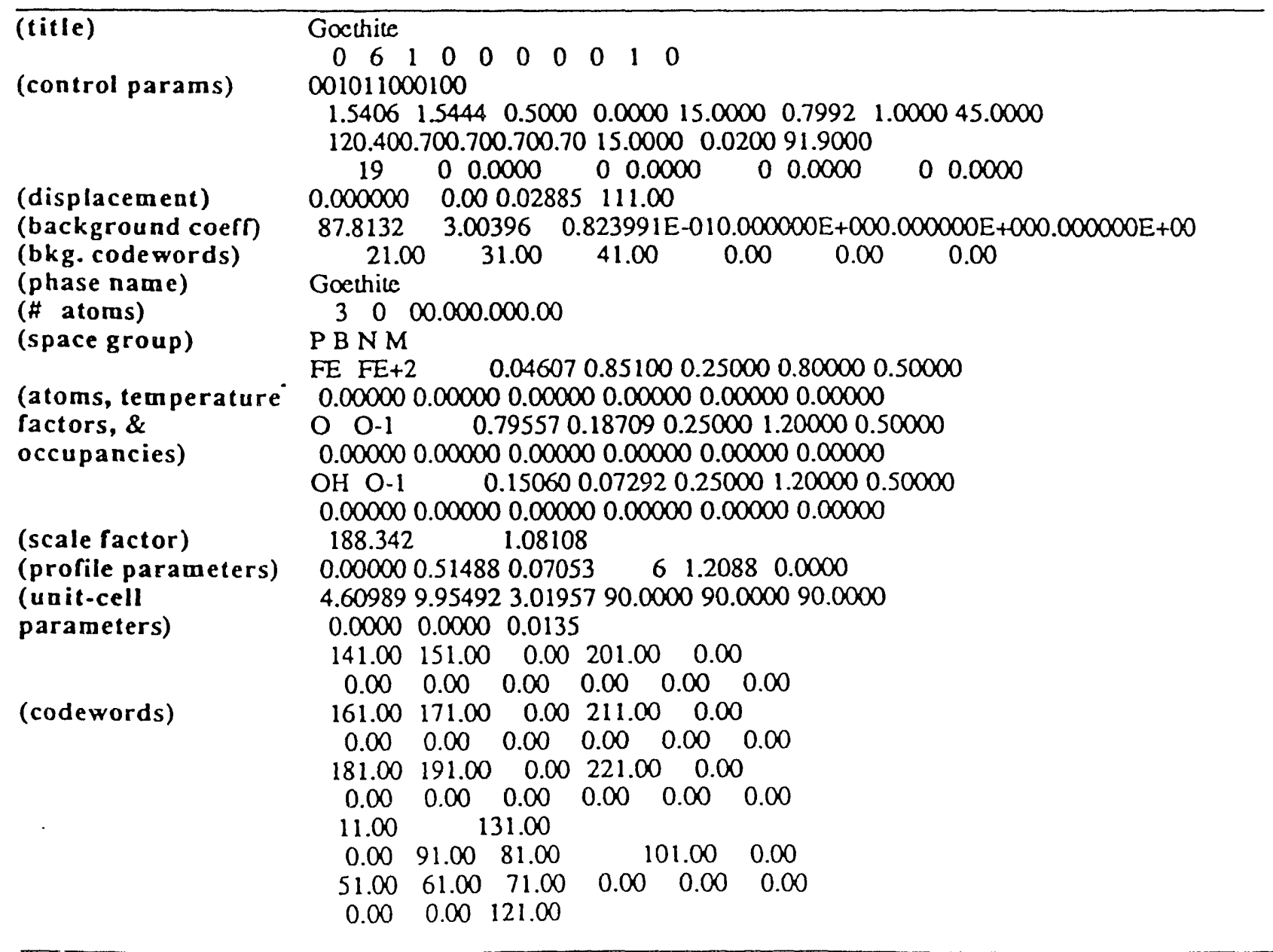


Bish

Once all profile-related parameters, unit-cell parameters, and atom positions have been stably refined, it should be possible to refine variably occupied sites if the scattering power of the occupants differ, e.g., $\mathrm{Fe}$ and $\mathrm{Mg}$ for X-rays. Site-occupancy refinement of atoms of similar scattering power, e.g., $\mathrm{Al}$ and $\mathrm{Si}$ with $\mathrm{X}$-rays, should not usually be attempted because unpredictable results will probably occur. If data are available over a wide angular range, it is usually possible to refine individual temperature factors, particularly if like atoms are constrained to have the same shifts. However, simultaneous refinement of temperature factors and site occupancies often yields unstable results due to correlations between the two. If partial structure solution is desired, e.g., determination of the location of an exchangeable cation in a zeolite, the recommendations in Post and Bish (1989) should be followed to avoid biasing the refinement.

The Rietveld method is more prone to yielding inaccurate refined structures than are singlecrystal methods, and it is always important to assess the reliability of a final structure. The weighted profile residual [Eq. (1)] provides a means of assessing the quality of the fit, but it does not allow assessment of the statistical significance of the refined structure. A variety of statistical parameters, including the Durbin-Watson $d$ statistic and the reduced $\chi^{2}$ statistic (see Post and Bish, 1989) have been proposed that allow one to obtain an idea of how significant a final result is. Irrespective of what $R_{w p}$ value is obtained, it is important to assess how crystal-chemically reasonable a final structure is. Generally, this involves a comparison of the observed bond distances and angles with published results from comparable structures. If observed bond distances deviate significantly from accepted values, the refined results should be suspect. In rare (?) cases, the refined structure may represent a false-minimum result, although poor and inaccurate structures usually result from various combinations of incorrect starting model, poor-quality data, incorrect refinement strategies, and uncorrected systematic errors.

When presenting results, it is important to provide all information pertinent to the refinement, including the type of profile function used, the value and type of preferred orientation correction, and the type of temperature factors (individual or overall) used. Observed, calculated, and difference curves should also be provided in plots, and it is essential to state whether background has been subtracted or not when using neutron powder diffraction data. Markers showing the calculated positions of possible reflections are often useful but are less so for complex patterns. The wavelength, scan range, count time per step, and temperature used in data collection should always be provided. Finally, in order to allow the reader to assess the quality of the refinement, both $R_{w p}$ and expected $R\left(R_{e x p}\right)$ value should be provided (in addition to one of the useful statistical parameters, $d$ or reduced $\chi^{2}$ ). $R_{\text {exp }}$ is essentially the minimum $R_{w p}$ that can be obtained and is one measure of how much information remains unresolved in the data after refinement.

\section{CONCLUSIONS}

Rietveld refrnement is clearly a versatile and powerful method that can be applied to solve a variety of problems in clay mineralogy if the user is aware of the appropriate sample limitations and the assumptions inherent to the method. The method can be applied to well-ordered clays and many other fine-grained minerals, such as many oxides and hydroxides, without significant assumptions and it can provide information that was previously beyond reach. This information includes accurate and precise unit-cell parameters, separation of crystallite-size and strain 
broadening effects, quantitative multicomponent analysis, partial structure solution, and full structure refinement. The method is particularly important for such materials because it allows analysis of very finely $c_{1}$ ystalline minerals that may be unavailable in single-crystal form.

The Rietveld method is limited in its application to disordered clays and clay minerals because it implicitly assumes the presence of Bragg diffraction effects only. Furthermore, it is very important to understand that two-dimensional diffraction effects cannot be approximated by broadening reflections using the profile shape functions typically available with the Rietveld method. Thus, a full three-dimensional refinement of such materials cannot be performed, but abbreviated analysis is still possible, including one-dimensional structure refinement which pre vidies a projection of all atoms onto $c$. Such an analysis can provide accurate and precise iniormation on layer repeat and can potentially provide information on site occupancies. Semirandom stacking sequences present an intermediate case, but their analysis will probably lead to a refined structure that is at least partially an artifact due to the presence of some two-dimensional diffraction effects.

\section{ACKNOWLEDGMENTS}

I am grateful to G. Guthrie for insightful comments on the manuscript and for assistance with figures. The manuscript has also benefitted significantly from comments by R. Reynolds and J. Walker.

\section{REFERENCES CITED}

Adarns, J. M. (1583) Hydrogen atom positions in kaolinite by neutron profile refinement: Clays \& Clay Minerals 3 , 352-356.

Adams, J. M. and Hewat, A. W. (1981) Hydrogen atom positions in dickite: Clays \& Clay Minerals 29, 316-319.

Banfield, J. F., Veblen, D. R., and Smith, D. J. (19.71) The identification of naturally occurring $\mathrm{TiO}_{2}(\mathrm{~B})$ by structure determinatirn using high-resolution electron microscopy, image simulations, and distance-least-squares refinement: American Mineralogist 76, 343-353.

Bish, D. L. (1989) Rietveld refinement of the kaolinite structure at 88, 294, and 573K: Clay Minerals Society 26th Annual Meeting, Abstracts, p. 17.

Bish, D. L. (1992) Structure building with Rietveld analysis: in Accuracy in Powder Diffraction II, Proceedings of the Intemational Conference May 26-29, 1992, NIST Special Publication $846,154-164$. 
Bish

Bish, D. L. (1993) Applications of Rietveld methods to clays and clay minerals: in CMS Workshop Lectures, Neutron Scattering Methods, F. Ross and J. Walker, eds., The Clay Minerals Society, in press.

Bish, D. L. (1994) Rietveld refinement of the kaolinite structure at $1.5 \mathrm{~K}$ : Clays \& Clay Minerals, in press.

Bish, D. L. and Ebinger, M. H. (1989) Rietveld refinement of synthetic goethite and Mnsubstituted goethite: in Proceedings of the 26th Annual Clay Minerals Society Meeting, 18.

Bish, D. L. and Howard, S. A. (1988) Quantitative phase analysis using the Rietveld method: Journal of Applied Crystallography 21, 86-91.

Bish, D. L. and Johnston, C. T. (1993) Rietveld refinement and Fourier-transform infrared spectroscopic study of the dickite structure at low temperature: Clays \& Clay Minerals 41, in press.

Bish, D. L. and Jones, R. C. (1991) Quantitative X-ray diffraction analysis of soils: Comparison of conventional curve-fitting and Rietveld full-pattern methods: in Proceedings of the 28th Annual Clay Minerals Society Meeting, 16.

Bish, D. L.. and Post, J. E. (1987) Refinement of the sepiolite structure and location of tunnel water molecules using the Rietveld method: in Proceedings of the 24th Annual Clay Minerals Society Meeting, 33.

Bish, D. L. and Post, J. E. (1993) Quantitative mineralogical analysis using the Rietveld fullpattern fitting method: American Mineralogist 78, in press.

Bish, D. L. and Reynolds, R. C., Jr. (1989) Sample preparation for X-ray diffraction: in Modern Powder Diffraction, D. L. Bish and J. E. Post, eds., Mineralogical Society of America, Washington, D. C., 73-99.

Bish, D. L. and Von Dreele, R. B. (1989) Rietveld refinement of non-hydrogen atomic positions in kaolinite: Clays \& Clay Minerals 37, 289-296.

Bish, D. L. and Von Dreele, R. B. (1990) The crystal structure of kaolinite including hydrogen atoms: in Proceedin ss of the 27th Annual Clay Minerais Society Meeting, 25.

Bish, D. L., Giese, R. F., Jr., and Costanzo, P. M. (1992) Crystal structure of the $8.4 \AA$ kaolinitehydrate complex: in Proceedings of the 29th Annual Clay Minerals Society Meeting. 
Brauner, K. and Preisinger, A. (1956) Struktur und Entstehung des Sepioliths: Miner. Petrogr. Mitt. 6, 120-140.

Buseck, P. R. and Veblen, D. R. (1988) Mineralogy: in High-Resolution Transmission Electron Microscopy and Associated Techniques, P. Buseck, J. Cowley, and L. Eyring, eds., Oxford Univ. Press, New York, 308-377.

Cagliotti, G., Paoletti, A., and Ricci, F. P. (1958) Choice of collimators for a crystal spectrometer for neutron diffraction: Nuclear Instrumentation 3, 223-228.

Catlow, C. R. A., Doherty, M., Price, G. D., Sanders, M. J., and Parker, S. C. (1986) Computer simulation studies in silicates: Materials Science Forum 7, 163-176.

Chung, F. H. (1974a) Quantitative interpretation of X-ray diffraction patterns of mixtures. I. Matrix-flushing method for quantitative multicomponent analysis: Journal of Applied Crystallography 7, 519-525.

Chung, F. H. (1974b) Quantitative interpretation of X-ray diffraction patterns of mixtures. II. Adiabatic principle of $\mathrm{X}$-ray diffraction analysis of mixtures: Journal of Applied Crystallography 7, 526-531.

Costanzo, P. M., Clemency, C. V., and Giese, R. F., Jr. (1980) Low-temperature synthesis of a $10 \AA$ hydrate of kaolinite using dimethylsulfoxide and ammonium fluoride: Clays \& Clay Minerals 28, 155-156.

Ebinger, M. H. and Bish, D. L. (1990) Rietveld refinement of goethite and Cr-goethite from X-ray powder data: in Proceedings of the 27th Annual Clay Minerals Society Meeting, 46.

Dollase, W. A. (1986) Correction of intensities for preferred orientation in powder diffractometry: Application of the March model: Journal of Applied Crystallography 19, 267-272.

Giese, R. F., Jr. (1982) Theoretical studies of the kaolin minerals: Electrostatic calculations: Bulletin Minéralogie 105, 417-424.

Giese, R. F. and Datta, P. (1973) Hydroxyl orientation in kaolinite, dickite, and nacrite: American Mineralogist 58, 471-479.

Guthrie, G. D. and Bish, D. L. (1991) Ionic modeling of the hydrogen sites in the kaolin polymorphs: in Proceedings of the 28th Annual Meeting of the Clay Minerals Society, Houston, Texas, p. 63 (abstract). 
Bish

Guthrie, G. D. and Bish, D. L. (1993) Ionic modeling of the hydrogen sites in the kaolin minerals: Clays \& Clay Minerals submitted.

Guthrie, G. D. and Veblen, D. R. (1990) Interpreting one-dimensional high-resolution transmission electron micrographs of sheet silicates by computer simulation: American Mineralogist 75, 276-288.

Joswig, W. and Drits, V. A. (1986) The orientation of the hydroxyl groups in dickite by X-ray diffraction: Neues Jahrbuch fur Mineralogie Monhatshefte 19-22.

Larson, A. C. and Von Dreele, R. B. (1988) GSAS. Generalized structure analysis system: Los Alamos National Laboratory Report LAUR 86-748, 150 pp.

Meier. W. M. and Villiger, H. (1969) Die Methode der Abstandsvergeinerung zur Bestimmung der Atomkoordinaten idealisierter Geruststrukturen: Zeitschrift fur Kristallographie 129, 411-423.

Nagy, B. and Bradley, W. F. (1955) Structure of sepiolite: American Mineralogist 40, 885-892.

O'Keefe, M. A. (1984) Electron image simulation: A complementary processing technique: in Electron Optical Systems, J. J. Hren, F. A. Lenz, E. Munro, and P. B. Sewell, eds., SEM Inc., AMF O'Hare, Chicago, IL, 209-220.

Post, J. E. and Bish, D. L. (1989) Rietveld refinement of crystal structures using powder X-ray diffraction data: In D. L. Bish and J. E. Post, Eds., Modern Powder Diffraction. Mineralogical Society of America Reviews in Mineralogy, 20, 277-308.

Post, J. E. and Veblen, D. R. (1990) Crystal structure determinations of synthetic sodium, magnesium, and potassium bimessite using TEM and the Rietveld method: American Mineralogist 75, 477-489.

Rakovan, J. F. and Guggenheim, S. (1991) Rietveld refinement of a IIb-2 clinochlore: in Proceedings of the 28th Annual Clay Minerals Society Meeting, 132.

Rautureau, M. and Tchoubar, C. (1974) Précisions concernant l'analyse structurale de la sépiolite par microdiffraction électronique: Compt. Rendus. Acad. Sc. Paris 278B, 25-28.

Rietveld, H. M. (1967) Line profiles of neutron powder-diffraction peaks for structure refinement: Acta Crystallographica 22, 151-152.

Rietveld, H. M. (1969) A profile refinement method for nuclear and magnetic structures: Journal of Applied Crystallography 2, 65-71. 
Sen Gupta, P. K., Schlemper, E. O., Johns, W. D., and Ross, F. (1984) Hydrogen positions in dickite: Clays \& Clay Minerals 32, 483-485.

Suitch, P. R. and Young, R. A. (1983) Atom positions in highly ordered kaolinite: Clays \& Clay Minerals 31, 357-366.

Veblen, D. R. (1985) High-resolution transmission electron microscopy: Chapter 4 in: Electron Microscopy in the Earth Sciences, J. C. White, ed., Mineralogical Assoc. Canada, 63-90.

Walker, J. R. and Bish, D. L. (1989) Rietveld refinement of IIb chlorite: in Proceedings of the 26th Annual Meeting of the Clay Minerals Society, 73.

Walker, J. R. and Bish, D. L. (1992) Application of Rietveld refinement techniques to a disordered Ib Mg-chamosite: Clays \& Clay Minerals 40, 319-322.

Wiles, D. B. and Young, R. A. (1981) A new computer program for Rietveld analysis of X-ray powder diffraction patterns: Journal of Applied Crystallography 14, 149-151.

Young, R. A. and Hewat, A. W. (1988) Verification of the triclinic crystal structure of kaolinite: Clays \& Clay Minerals 36, 225-232.

Young, R. A. and Wiles, D. B. (1982) Profile shape functions in Rietveld refinements: Journal of Applied Crystallography 15, 430-438. 

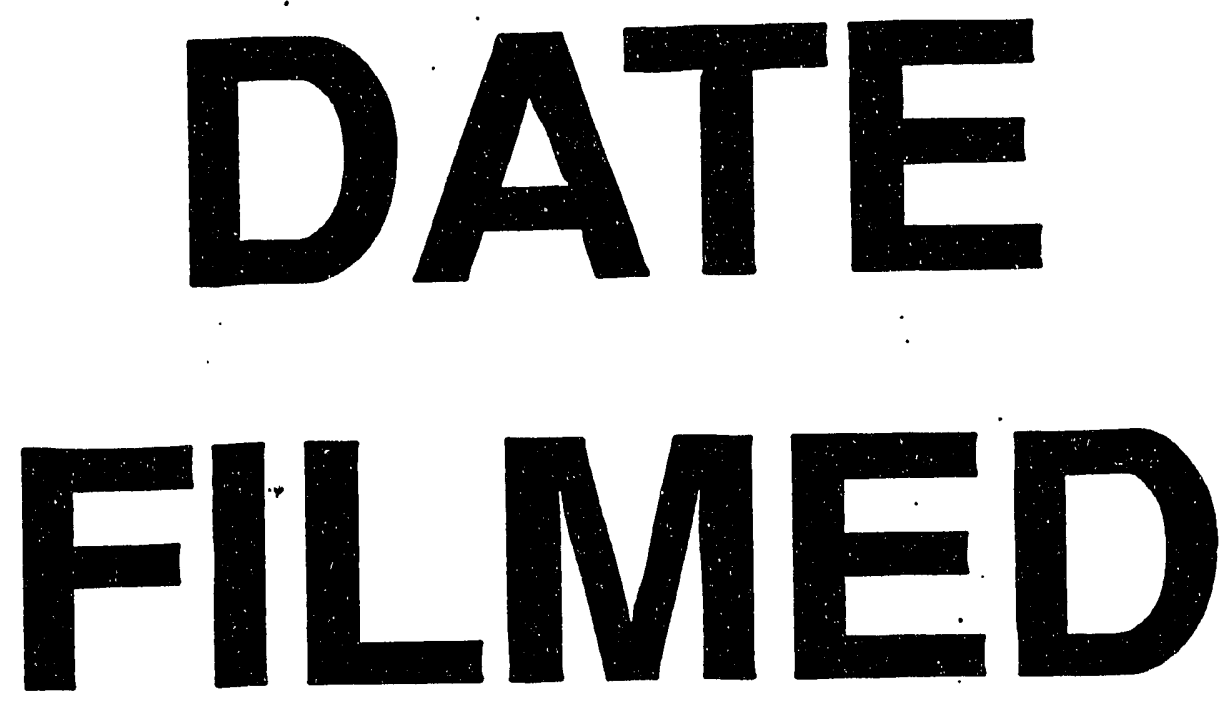

$12 / 7 / 93$
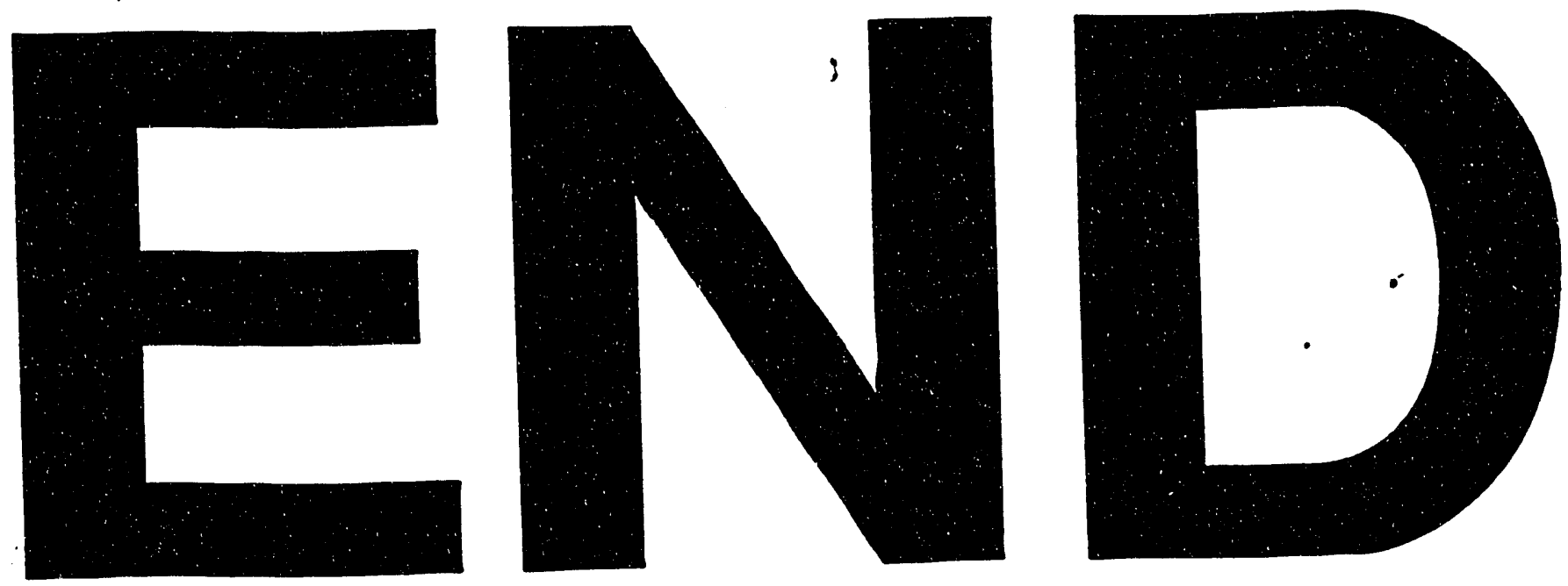
